\title{
DISCRETE SPECTRUM OF CRANKED, BRANCHING, AND PERIODIC WAVEGUIDES
}

\author{
S. A. NAZAROV
}

\begin{abstract}
The variational method is applied to the study of the spectrum of the Laplace operator with mixed boundary conditions and with Dirichlet conditions in planar or multidimensional domains (waveguides) with cylindrical or periodic exits to infinity. The planar waveguides of constant width are discussed completely, such as cranked, broken, smoothly bent, or branching waveguides. For them, the existence of eigenvalues below the continuous spectrum threshold is established. A similar result is obtained for the multidimensional cranked and branching waveguides, and also for some periodic ones. Several open questions are stated; in particular, they concern problems with Neumann boundary conditions, full multiplicity of the discrete spectrum, and planar waveguides with piecewise constant boundary.
\end{abstract}

\section{$\S 1$. INTRODUCTION}

1. Setting of two-dimensional problems. Consider the Helmholtz equation

$$
-\Delta_{x} u(x)=\lambda u(x), \quad x \in \Omega,
$$

in a domain $\Omega \subset \mathbb{R}^{2}$ with several exits to infinity in the form of half-strips $\Pi_{1}, \ldots, \Pi_{N}$ of unit width. A simplest domain of this sort is depicted in Figure 1: this is a "cranked" waveguide; for $N \geq 3$ the domain $\Omega$ is a "branching", in particular, "cross-like" waveguide (see Figures $2 \mathrm{a}$ and $3 \mathrm{a}$, respectively). Equation (1.1) involves the Laplace operator $\Delta_{x}$ written in a Cartesian coordinate system $x$, and the spectral parameter $\lambda$. The domain $\Omega$ is composed of "pointed" half-strips as is shown in Figure $2 \mathrm{~b}$, and in some situations (but not always: cf. Figure 4 , a and b) $\Omega$ can be given as follows:

$$
\Omega=\{x: \operatorname{dist}(x, L)<1 / 2\},
$$

where $L$ is the union of rays $L_{1}, \ldots, L_{N}$ emanating from the origin $\mathcal{O}$ of the Cartesian coordinate system $x=\left(x_{1}, x_{2}\right)$ and making angles $\alpha_{1}, \ldots, \alpha_{N} \in[0,2 \pi)$ with the positive semiaxis $\left\{x: x_{2}=0, x_{1}>0\right\}=L_{1}$,

$$
0=\alpha_{1}<\alpha_{2}<\cdots<\alpha_{N-1}<\alpha_{N}<2 \pi .
$$

It is easy to check that if the angles between the axes of any two neighboring half-strips do not exceed $\pi$, then the definition (1.2) fits.

In $\S 2$ we study the Dirichlet problem

$$
u(x)=0, \quad x \in \partial \Omega,
$$

for equation (1.1), and also the mixed boundary value problem

$$
u(x)=0, \quad x \in \Gamma_{D}, \quad \partial_{\nu} u(x)=0, \quad x \in \Gamma_{N},
$$

2010 Mathematics Subject Classification. Primary 35Q60.

Key words and phrases. Eigenvalues, discrete spectrum, Laplace operator, cranked, branching, and periodic waveguides.

Supported by RFBR (grant no. 09-01-00759). 

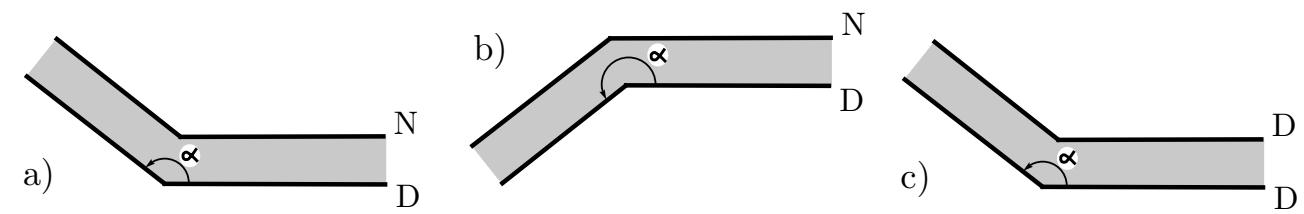

FiguRE 1. Cranked waveguides with mixed boundary conditions (a and b) and Dirichlet condition (c).

for $N=2$ (a cranked waveguide). Here $\partial_{\nu}$ is the derivative along the outward normal to the part $\Gamma_{N}$ of $\partial \Omega$, and $\Gamma_{D}=\partial \Omega \backslash \Gamma_{N}$. It is checked that if $\alpha_{2}<\pi$ (Figure 1a), then the discrete spectrum of problem (1.1), (1.5) is empty, but if $\alpha_{2}>\pi$, then this spectrum has at least one eigenvalue. Also, in Theorems 2.3 and 2.5 we show that the discrete spectrum $\sigma_{d}$ of problem (1.1), (1.4) is always nonempty.

In $\S 2$ we discuss also some other forms of planar waveguides of constant width (see Figures 5, 6, and 7). In Subsection 6 of $\S 2$ we carry some of the results over to the case of planar waveguides of piecewise-constant width. In $\S 3$ we consider multidimensional waveguides, and in $\S 4$ we treat periodic ones. In all cases we deduce conditions ensuring the existence of eigenvalues in the discrete spectrum; for this, we apply a variational method collected from several sources and complemented and stated in a final form in [2, 3, and also a new generalization of that method for waveguides with variable cross section. We mention the publications [1] and [4]-16], where some other methods for tracing the discrete and the point spectrum were applied, and also for waveguides of other forms. The survey paper [17] makes it possible to extend the list of references substantially.

It should be emphasized that, in any of the two-dimensional problems we consider, the Dirichlet boundary conditions are posed on the parts of the boundary that go to infinity. I know of no example of a planar cranked or branching waveguide for which the Neumann problem has an eigenvalue (now belonging to the point spectrum). Only for some symmetric multidimensional waveguides such eigenvalues can be found within our approach combined with the method (see [7) of posing artificial boundary conditions (see Subsection 5 in $\S 3$ ). The techniques used in the present paper are not adjusted to calculation of the full multiplicity of the discrete spectrum, so that even the question as to whether the eigenvalue $\lambda \in\left(0, \lambda_{\dagger}\right)$ that we have found is unique remains, as a rule, open. As an exception, we mention Theorem 2.2, showing that $\sigma_{d}=\varnothing$ in problem (1.1), (1.5) for the waveguides as in Figure 1a, and also Example 2.1 and Remark 3.1 where we check that the discrete spectrum for the waveguides in Figures 8a and 3a can consist of at most one element. However, the particular methods we use can we applied also to other specific problems.

2. The variational method for the search of eigenvalues below the threshold. The variational setting of problem (1.1), (1.5) appeals to the integral identity

$$
\left(\nabla_{x} u, \nabla_{x} v\right)_{\Omega}=\lambda(u, v)_{\Omega}, \quad v \in \stackrel{\circ}{H}^{1}\left(\Omega ; \Gamma_{D}\right)
$$

(see [18]), where $\nabla_{x}=\operatorname{grad},(,)_{\Omega}$ is the natural scalar product in the Lebesgue space $L_{2}(\Omega)$, and $\stackrel{\circ}{H}^{1}\left(\Omega ; \Gamma_{D}\right)$ is the subspace in the Sobolev space $H^{1}(\Omega)$ formed by the functions subject to the first boundary condition in (1.5). In the case of the Dirichlet conditions (1.4) we have $\Gamma_{D}=\partial \Omega$, and the notation $\stackrel{\circ}{H}^{1}(\Omega ; \partial \Omega)$ will be abbreviated to $\stackrel{\circ}{H}^{1}(\Omega)$. 

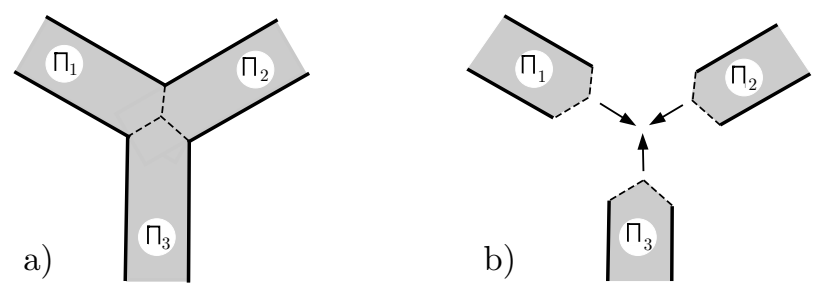

FiguRE 2. Branching waveguides.
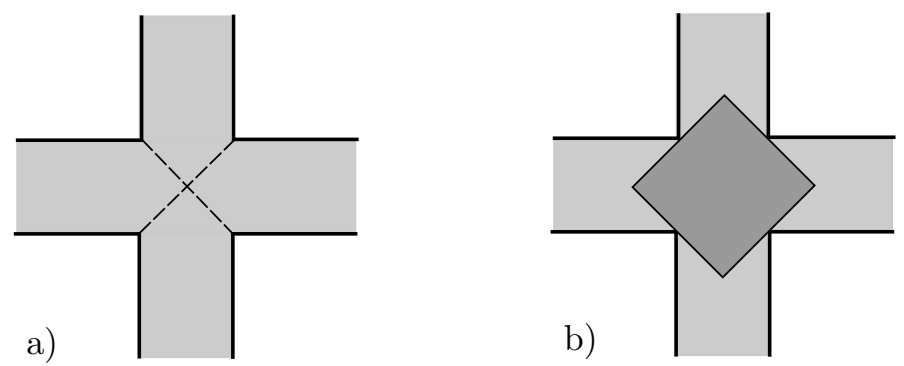

Figure 3. Cross-like waveguides.

a)

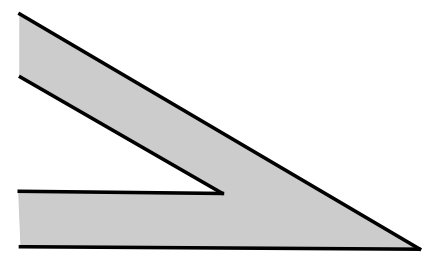

b)

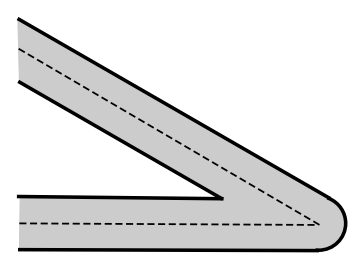

Figure 4. Inadequacy of the definition (1.2).
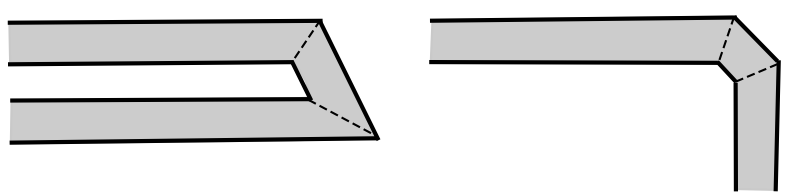

Figure 5. Broken waveguides.

a)

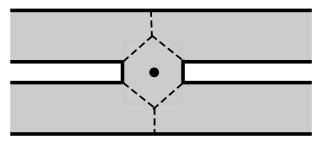

b)

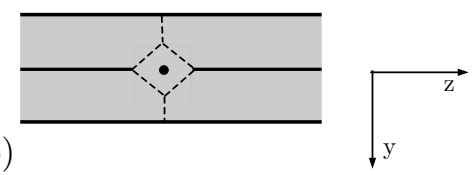

Figure 6. Connection of straight waveguides with bridges. 
a)

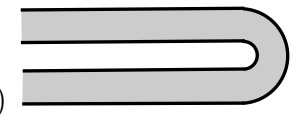

b)

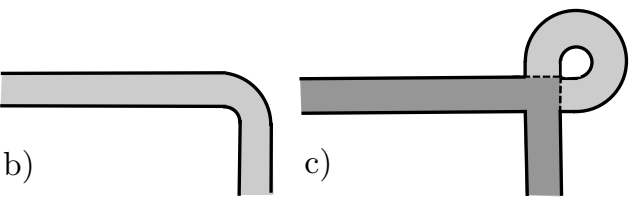

FiguRe 7. Smoothly bent waveguides.

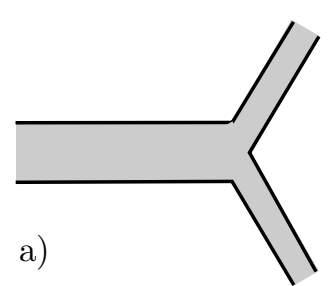

FiguRE 8. Planar waveguides with piecewise-constant width.

Due to the Dirichlet condition and the cylindrical nature of our domain near infinity, we have

$$
\left\|u ; L_{2}(\Omega)\right\| \leq c_{\Omega}\left\|\nabla_{x} u ; L_{2}(\Omega)\right\|, \quad u \in \stackrel{\circ}{H}^{1}\left(\Omega ; \Gamma_{D}\right),
$$

Hence, the left-hand side of (1.6) is a scalar product in the Sobolev space $\stackrel{\circ}{H}^{1}\left(\Omega ; \Gamma_{D}\right)$. Thus, the variational problem (1.6) gives rise (see [19. Chapter 10]) to an unbounded, positive, and selfadjoint operator $A$ in the Hilbert space $L_{2}(\Omega)$.

As is known (and easy to check), for a planar domain $\Omega$ going to infinity along halfstrips of unit width, the continuous spectrum

$$
\sigma_{c}=\left[\lambda_{\dagger},+\infty\right)
$$

of the operator $A$ has the threshold

$$
\lambda_{\dagger}= \begin{cases}\pi^{2} / 4 & \text { for conditions (1.5) } \\ \pi^{2} & \text { for conditions (1.4). }\end{cases}
$$

If $\lambda \geq \lambda_{\dagger}$, the homogeneous problem in the strip

$$
\Pi_{0}=\left\{x=\left(x_{1}, x_{2}\right)=(y, z): 0<y<1, z \in \mathbb{R}\right\}
$$

admits an oscillating (or constant in the variable $z$ for $\lambda=\lambda_{\dagger}$ ) solution 1

$$
\exp \left( \pm i\left(\lambda-\lambda_{\dagger}\right)^{1 / 2} z\right) U_{\dagger}(y)
$$

with the help of which we can easily build a Weil singular sequence for the operator $A$ at the point $\lambda$ (see, e.g., [19, $\S 9.1]$ ). In (1.10), $i$ is the imaginary unit, and

$$
U_{\dagger}(y)= \begin{cases}\sin (\pi y / 2) & \text { in the case of (1.5), } \\ \sin (\pi y) & \text { in the case of (1.4). }\end{cases}
$$

Below the threshold $\lambda_{\dagger}$, the operator $A$ can have a discrete spectrum $\sigma_{d} \subset\left(0, \lambda_{\dagger}\right)$. The continuous spectrum (1.7) coincides with the essential spectrum, so that the entire spectrum of $A$ is the union of two disjoint sets $\sigma_{d}$ and $\sigma_{c}$.

\footnotetext{
${ }^{1}$ A running (or stationary) wave.
} 


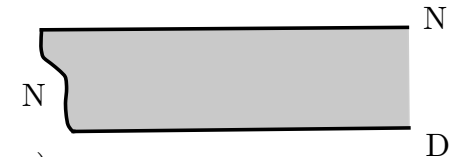

a)

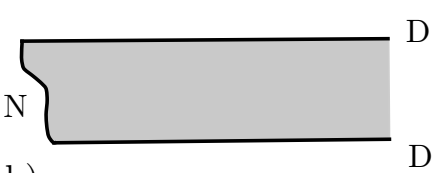

b)

Figure 9. A half-strip with curvilinear end.

We pass to the description of our variational method, which consists of several specific technical tricks. In the half-strip

$$
\Pi^{H}=\{x=(y, z): y \in(0,1),-H(y)<z\}
$$

with curvilinear end, we consider the mixed boundary-value problem

$$
\begin{aligned}
& -\Delta_{x} v(x)=\mu v(x), \quad x \in \Pi^{h}, \\
& v(x)=0, \quad x \in \varpi_{D}^{H}, \quad \partial_{\nu} v(x)=0, \quad x \in \varpi_{N}^{H},
\end{aligned}
$$

which gives rise (see [19, §9.1]) to an operator $A^{H}$ in the Hilbert space $L_{2}\left(\Pi^{H}\right)$. Here $H$ is a nonnegative, continuous, and piecewise smooth function, and

$$
\varpi_{D}^{H}=\{0\} \times(-H(0),+\infty)
$$

or

$$
\varpi_{D}^{H}=(\{0\} \times(-H(0),+\infty)) \cup(\{1\} \times(-H(1),+\infty)),
$$

where $\varpi_{N}^{H}=\partial \Pi^{H} \backslash \overline{\varpi_{D}^{H}}$ (see Figure 9, a and b). In the situation (1.14), the Dirichlet conditions are posed on the lower lateral side of the half-strip, while in the case of (1.15) they are posed on both lateral sides.

In the paper 20, the following theorem was established with the help of the variational method for the search of the discrete spectrum, as formulated in [2, 3] (see also [21, 14, 15]); we repeat the verification of this theorem for the reader's convenience.

Theorem 1.1. 1) The discrete spectrum of the operator $A^{H}$ of problem (1.13) is nonempty provided that

$$
\int_{0}^{1} H(y) \cos (\pi y) d y<0
$$

in the case of (1.14) and, in the case of (1.15), provided that

$$
\int_{0}^{1} H(y) \cos (2 \pi y) d y<0 .
$$

2) If the integral on the left in (1.16) (in (1.17) ) is zero, but the normal derivative of the function (1.11) is not identically zero on the art2

$$
\varpi_{0}^{H}=\{x=(y, z): y \in(0,1), z=-H(y)\}
$$

then the discrete spectrum of $A^{H}$ is still nonempty.

\footnotetext{
${ }^{2}$ The relation $\partial_{\nu} U_{\dagger}=0$ a.e. on the set (1.18) is possible only if $H(y)=$ const (see Remark 1.1 below).
} 
Proof. 1) By [19, §9.2], the lower bound $\underline{\sigma}^{H}$ of the spectrum $\sigma^{H}$ of $A^{H}$ is calculated by the formula 3

$$
\underline{\sigma}^{H}=\inf _{u \in \dot{H}^{1}\left(\Pi^{H} ; \varpi_{D}^{H}\right) \backslash\{0\}} \frac{\left\|\nabla_{x} u ; L_{2}\left(\Pi^{H}\right)\right\|^{2}}{\left\|u ; L_{2}\left(\Pi^{H}\right)\right\|^{2}} .
$$

Since $\sigma_{d}^{H}=\sigma^{H} \backslash \sigma_{c}^{H}$ and $\sigma_{c}^{H}=\left[\lambda_{\dagger},+\infty\right)$, in the case where $\underline{\sigma}^{H}<\lambda_{\dagger}$ the discrete spectrum $\sigma_{d}^{H}$ cannot be empty, and $\lambda_{1}^{H}=\underline{\sigma}^{H}$ is the lowest eigenvalue of the operator $A^{H}$.

We plug the test function

$$
u^{\varepsilon}(x)= \begin{cases}U_{\dagger}(y) & \text { if } z<0 \\ \exp (-\varepsilon z) U_{\dagger}(y) & \text { if } z \geq 0\end{cases}
$$

in the right-hand side of (1.19), where $\varepsilon$ is a small positive parameter. Due to the exponential factor, the function (1.20) falls into the space $\stackrel{\circ}{H}^{1}\left(\Pi^{H} ; \varpi_{D}^{H}\right)$. The further calculations will be done in the situation (1.15) (the case of (1.14) requires only elementary modifications). We have

$$
\begin{aligned}
\left\|u^{\varepsilon} ; L_{2}\left(\Pi^{H}\right)\right\|^{2} & =\left\|u^{\varepsilon} ; L_{2}\left(\Pi_{+}\right)\right\|^{2}+\left\|u^{\varepsilon} ; L_{2}\left(\Pi^{H} \backslash \Pi_{+}\right)\right\|^{2} \\
& =\int_{0}^{1} \int_{0}^{\infty} \exp (-2 \varepsilon z)\left|U_{\dagger}(y)\right|^{2} d z d y+\int_{0}^{1} \int_{-H(y)}^{0}\left|U_{\dagger}(y)\right|^{2} d z d y \\
& =\frac{1}{4 \varepsilon}+\int_{0}^{1} H(y)(\sin (\pi y))^{2} d y, \\
\left\|\nabla_{x} u^{\varepsilon} ; L_{2}\left(\Pi^{H}\right)\right\|^{2} & =\frac{\pi^{2}}{4 \varepsilon}+\pi^{2} \int_{0}^{1} H(y)(\cos (\pi y))^{2} d y+\frac{\varepsilon}{4} .
\end{aligned}
$$

Consequently,

$$
\begin{aligned}
\underline{\sigma}^{H} \leq \frac{\left\|\nabla_{x} u^{\varepsilon} ; L_{2}\left(\Pi^{H}\right)\right\|^{2}}{\left\|u^{\varepsilon} ; L_{2}\left(\Pi^{H}\right)\right\|^{2}} & \leq \frac{1+4 \varepsilon \int_{0}^{1} H(y)(\cos (\pi y))^{2} d y+c \varepsilon^{2}}{1+4 \varepsilon \int_{0}^{1} H(y)(\sin (\pi y))^{2} d y} \\
& \leq \pi^{2}\left(1+4 \varepsilon \int_{0}^{1} H(y) \cos (2 \pi y) d y+C_{H} \varepsilon^{2}\right) .
\end{aligned}
$$

Thus, under condition (1.17), the right-hand side of (1.22) can be made less than $\lambda_{\dagger}=\pi^{2}$ by choosing the small number $\varepsilon>0$ appropriately. In other words, $\underline{\sigma}^{H}<\pi^{2}$, and the discrete spectrum includes the eigenvalue $\underline{\sigma}^{H}$.

2) As before, we restrict ourselves to the situation (1.15); then, by the assumption of part 2 of the theorem, we have

$$
\int_{0}^{1} H(y) \cos (2 \pi y) d y=0 .
$$

For the role of the test function, we take the sum $u^{\varepsilon}+\sqrt{\varepsilon} v$, where $u^{\varepsilon}$ is given by (1.20), and $v$ is an arbitrary smooth function supported on a neighborhood of the end (1.18) of the half-strip. If the discrete spectrum is empty, so that

$$
\underline{\sigma}^{H}=\lambda_{\dagger}
$$

\footnotetext{
${ }^{3}$ We draw the reader's attention to the convenience of the theory presented in [19, Chapter 10]: the infimum is calculated on the "energy" space $\stackrel{\circ}{H}^{1}\left(\Pi^{H} ; \varpi_{D}^{H}\right)$, rather than on the domain of the operator $A^{H}$; due to the possible singularities of the boundary, this domain may fail to coincide with $H^{2}\left(\Pi^{H}\right) \cap$ $\stackrel{\circ}{H}^{1}\left(\Pi^{H} ; \varpi_{D}^{H}\right)$.
} 

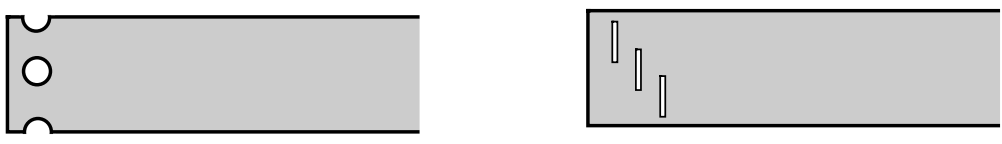

FIgURE 10. Half-strips with holes and cracks.

a) $\mathrm{k}>0$

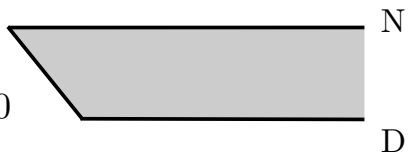

b) $\mathrm{k}<0$

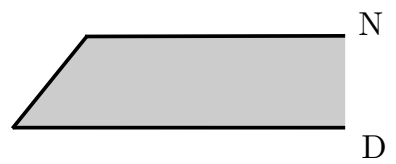

FiguRE 11. Half-strips with slanted ends.

(cf. (1.8) ), then, by formula (1.19), we have

$$
\left\|u^{\varepsilon}+\sqrt{\varepsilon} v ; L_{2}\left(\Pi^{H}\right)\right\| \leq \pi^{-2}\left\|\nabla_{x} u^{\varepsilon}+\sqrt{\varepsilon} \nabla_{x} v ; L_{2}\left(\Pi^{H}\right)\right\| .
$$

Using the calculations (1.21), we find that the two sides of (1.25) are equal, respectively, to the expressions

$$
\begin{aligned}
& \frac{1}{4 \varepsilon}+\int_{0}^{1} H(y)(\sin (\pi y))^{2} d y+2 \sqrt{\varepsilon} \int_{\Pi^{H}} U_{\dagger}(y) v(x) d x+O(\varepsilon), \\
& \frac{1}{\pi^{2}}\left(\frac{\pi^{2}}{4 \varepsilon}+\pi^{2} \int_{0}^{1} H(y)(\cos (\pi y))^{2} d y+2 \sqrt{\varepsilon} \int_{\Pi^{H}} \partial_{y} U_{\dagger}(y) \partial_{y} v(x) d x\right)+O(\varepsilon) .
\end{aligned}
$$

Both integrals over the set $\Pi^{H}$ in (1.26) converge because the support supp $v$ is compact. Then, by (1.23) and (1.26), inequality (1.25) takes the form

$$
2 \sqrt{\varepsilon} \int_{\Pi^{H}} U_{\dagger}(y) v(x) d x \leq \frac{1}{\pi^{2}} 2 \sqrt{\varepsilon} \int_{\Pi^{H}} \partial_{y} U_{\dagger}(y) \partial_{y} v(x) d x+C \varepsilon .
$$

Next we integrate by parts and use the simple equation $-\partial_{y}^{2} U_{\dagger}(y)=\pi^{2} U_{\dagger}(y)$ to reshape this inequality to

$$
-2 \sqrt{\varepsilon} \int_{\varpi_{0}^{H}} v(x) \partial_{\nu} U_{\dagger}(y) d s_{x} \leq C \varepsilon .
$$

If $\mp \partial_{\nu} U_{\dagger}\left(y^{0}\right)>0$ at a point $\left(y^{0},-H\left(y^{0}\right)\right) \in \varpi_{0}^{H}$, then, for a function $\pm v$ with small support positive at that point, the integral in (1.27) becomes positive, and relation (1.27) itself becomes absurd for small $\varepsilon>0$. Thus, (1.24) cannot be true, and the discrete spectrum is nonempty.

Remark 1.1. Theorem 1.1 admits simple generalizations to the case of semibounded sets (e.g., a half-strip with holes as in Figure 10a). The condition $\partial_{\nu} U_{\dagger} \not \equiv 0$ on $\varpi_{0}^{H}$ is only violated for the half-strip with right end itself, or for such a half-strip with a family of cracks parallel to its end (see Figure 10b).

Example 1.1. Let $H(y)=k y$ (Figure 11). In the case of (1.14) we have

$$
\int_{0}^{1} H(y) \cos (\pi y) d y=\left.\frac{k}{\pi}\left(y \sin (\pi y)+\frac{1}{\pi} \cos (\pi y)\right)\right|_{0} ^{1}=-\frac{k}{\pi^{2}} .
$$

Therefore, if the coefficient $k$ (see Figure 11) is positive, Theorem 1.1 guarantees that $\sigma_{d}^{H} \neq \varnothing$. In Remark 2.1 we shall explain why $\sigma_{d}^{H}=\varnothing$ if $k \leq 0$ (Figures $11 \mathrm{~b}$ and $12 \mathrm{~d}$ ). 
a) $\mathrm{k}_{1}<\mathrm{k}_{0}$

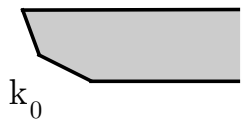

c) $\mathrm{k}_{0}=\mathrm{k}_{1} \neq 0$ b) $\mathrm{k}_{0}<\mathrm{k}_{1}$

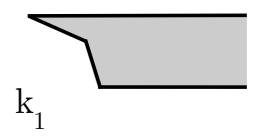

d) $\mathrm{k}_{0}=\mathrm{k}_{1}=0$

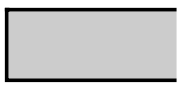

FIGURE 12. Half-strips with pointed (a and b), slanted (c), and right (d) ends.

Example 1.2. Let $t \in(0,1)$, and let, in accordance with Figure 12, the profile function be given by the formulas

$$
H(y)= \begin{cases}k_{0} y & \text { if } y \in(0, t) \\ k_{0} t+k_{1}(y-t) & \text { if } y \in(t, 1)\end{cases}
$$

In the case of (1.15), where the Dirichlet condition is posed on both lateral sides of the half-strip, we have

$$
\int_{0}^{1} H(y) \cos (2 \pi y) d y=\frac{1}{4 \pi^{2}}\left(k_{1}-k_{0}\right)(1-\cos (2 \pi t)) .
$$

If $k_{0}>k_{1}$ (Figure 12a), then the last factor is positive, so that condition (1.17) is satisfied, and the discrete spectrum is nonempty. If $k_{0}=k_{1}$, i.e., the integral (1.29) is equal to zero, but $k_{0} \neq 0$ (Figures 12c and 11a), then Theorem 1.1(2) again ensures that the discrete spectrum $\sigma_{d}^{H}$ is nonempty. Finally, if $k_{0}<k_{1}$ (Figure 12b), then Theorem 1.1 gives no information about the discrete spectrum.

Remark 1.2. In accordance with the arguments in Example 1.2, any slanted cut splits the half-strip into two semibounded waveguides such that a nonempty discrete spectrum emerges for the mixed boundary-value problem with Dirichlet conditions on the lateral sides of slanted half-strips and Neumann conditions on their ends.

3. Description of known and new results. As has been mentioned, Theorem 1.1 is borrowed from the paper 20. The claims about the existence of eigenvalues of the Dirichlet problem (1.1), (1.4) below the threshold $\lambda_{\dagger}=\pi^{2}$ of the continuous spectrum are known (see [22, 23]) for the domains as in Figures 3a and 1c (see also Theorems 2.5] and 2.3 respectively). Note that the fact that the discrete spectrum $\sigma_{d}$ is nonempty in the case of a cross-like waveguide is a consequence of a similar property of the spectrum for the cranked waveguide with the angle $\alpha_{2}$ equal to $\pi / 2$ (see (1.3) and Remark 2.2). This makes it possible to extend the claims in question to some waveguides with piecewiseconstant width (see Subsection 6 in $\S 2$ ). We mention curious observations made in [23. in connection with the result of [22]: first, in the union $\Omega$ of two mutually orthogonal strips of unit width, we can place the square $Q_{\sqrt{2}}$ with side $\sqrt{2}$ (see the deeply pointed square in Figure $3 \mathrm{~b}$ ), and the first eigenvalue of the Dirichlet problem for $Q_{\sqrt{2}}$ is equal to $2(\pi / \sqrt{2})^{2}=\pi^{2}$; second, for any bounded subdomain $\Omega_{\bullet} \supsetneqq Q_{\sqrt{2}}$ of $\Omega$, the first eigenvalue lies in the interval $\left(0, \pi^{2}\right)$; and third, since this interval does not intersect the continuous spectrum $\sigma_{c}=\left[\pi^{2}, \infty\right)$ (cf. formulas (1.7), (1.8) ) of the cross-like waveguide, such a waveguide has an eigenvalue $\lambda \in\left(0, \pi^{2}\right)$ by the minimal principle (1.19). It should be emphasized that, generally speaking, no similar simple arguments are a success for the planar waveguides with piecewise constant width studied in $\S 3$ (cf. Remark 2.3 and Example 2.1). 
Yet another series of known results on the existence of eigenvalues pertains to the case of smoothly bent waveguides depicted schematically in Figure 7, a and b (see the paper [1, where not only planar, but also 3-dimensional waveguides with constant cross sections were studied). In Theorem 2.4 we give a proof different from the original one (see 11) and based on the variational method mentioned above. Also, this method allows us to find eigenvalues on the interval $\left(0, \pi^{2}\right)$ in the case of broken waveguides (Figure 5).

In $\S 2$ below, we consecutively consider the three cranked waveguides as in Figure 1, and then the broken (Figure 5), smoothly bent (Figure 7), and branching (Figures 2, 3, and 6) waveguides. Also we discuss some noncentered waveguides (Subsection 5 in $\S 2$ ) and waveguides with piecewise-constant width. In $\S 3$ we apply the same techniques to study cranked and branching waveguides, and in $\S 4$ the variational method is generalized to cover the case of periodic waveguides. It should be emphasized that in the last case the results become somewhat conditional, because the eigenfunction of the model problem on the periodicity cell that corresponds to the first eigenvalue depends on all $n$ spatial variables. Subsection 5 of $\S 3$ deserves special mention: here we use the method of [7] to investigate the point spectrum (the eigenvalues embedded in the continuous spectrum) for the Neumann problem in symmetrical many-dimensional waveguides, and also present examples of planar branching waveguides for which the intersection of the point and the continuous spectrum is nonempty.

\section{§2. TWO-DIMENSIONAL WAVEGUIDES}

1. A cranked waveguide; the mixed boundary-value problem for $\alpha_{2} \in(\pi, 2 \pi)$. The bisector of the obtuse angle $2 \pi-\alpha_{2}$ with the vertex $P$ (see Figure 13) splits our cranked waveguide, which now is a broken strip $\Omega$ as in Figure 1b, into two slanted half-strips $\Pi_{p}, p=1,2$. In the local coordinates $\left(y_{p}, z_{p}\right)$ the half-strips $\Pi_{p}$ take the form (1.12) with one and the same profile function $H\left(y_{p}\right)=k y_{p}$ and the coefficient $k=\tan \left(\alpha_{2} / 2\right)<0$. Since the test function

$$
u^{\varepsilon}(x)= \begin{cases}U_{\dagger}\left(y_{p}\right) & \text { if } x \in \Pi_{p}, z_{p}<0 \\ \exp \left(-\varepsilon z_{p}\right) U_{\dagger}\left(y_{p}\right) & \text { if } x \in \Pi_{p}, z_{p}>0, p=1,2,\end{cases}
$$

(cf. the definition (1.20) ) turns out to be continuous everywhere, including the set where the half-strips meet, the claim below can be verified along the lines of the proof of Theorem 1.1 (see the calculations in Example 1.1).

Theorem 2.1. The operator $A$ of the mixed boundary-value problem (1.1), (1.5) in a cranked waveguide $\Omega$ as depicted in Figures 13 and $1 \mathrm{~b}$ with the angle $\alpha_{2} \in(\pi, 2 \pi)$ measured between the two rays carrying the Dirichlet conditions, always has an eigenvalue on the interval $\left(0, \pi^{2} / 4\right)$, i.e., the discrete spectrum $\sigma_{d}$ of the operator $A$ is nonempty.

2. A cranked waveguide; the mixed boundary-value problem for $\alpha_{2} \in(0, \pi)$. If $\alpha_{2} \in(0, \pi)$, then $k=\tan \left(\alpha_{2} / 2\right)>0$ in the notation as above (cf. Figure 14). Hence, condition (1.16) is violated, and Theorem 1.1 says nothing about the properties of the discrete spectrum. Actually, we have $\sigma_{d}=\varnothing$; to verify this we shall need an auxiliary claim.

Lemma 2.1. Consider a right triangle $\vartheta^{h}$ and pose the Neumann boundary conditions on the hypotenuse and on the leg of unit length, while on the other leg $\tau^{h}$ of length $h$ we pose the Dirichlet condition. Then the eigenvalue $\beta_{1}^{h}$ of such a mixed boundary-value problem for the Laplace operator satisfies the inequalities

$$
\frac{\pi^{2}}{4}<\beta_{1}^{h}<\beta_{1}^{0}
$$




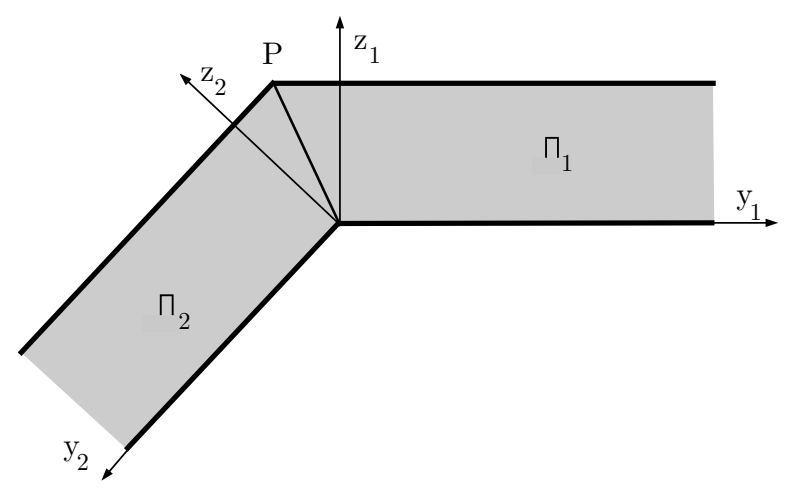

FIGURE 13. A cranked waveguide.

where $\beta_{1}^{0} \approx 2.4048 \ldots$ is the first eigenvalue of the Dirichlet problem for the Laplace operator in the unit disk (see, e.g., [24]).

Proof. The function $(0,+\infty) \ni h \mapsto \beta_{1}^{h}$ is monotone decreasing. To check this, we apply the minimal principle

$$
\beta_{1}^{h}=\inf _{w \in \dot{H}^{1}\left(\vartheta^{h} ; \tau^{h}\right)} \frac{\left\|\nabla_{x} w ; L_{2}\left(\vartheta^{h}\right)\right\|^{2}}{\left\|w ; L_{2}\left(\vartheta^{h}\right)\right\|^{2}} .
$$

In formula (2.3) with a perturbed parameter $h+\Delta h>h$, we substitute the test function

$$
w(y, z)=w_{1}^{h}\left(h(h+\Delta h)^{-1} y, z\right),
$$

belonging to the space $\stackrel{\circ}{H}^{1}\left(\vartheta^{h+\Delta h} ; \tau^{h+\Delta h}\right)$ and built starting with the first eigenfunction of the problem in the triangle $\vartheta^{h}$. Dilating the coordinate $y$, we obtain

$$
\begin{aligned}
\beta_{1}^{h+\Delta h} & \leq\left\|w ; L_{2}\left(\vartheta^{h+\Delta h}\right)\right\|^{-2}\left\|\nabla_{x} w ; L_{2}\left(\vartheta^{h+\Delta h}\right)\right\|^{2} \\
& =\left\|w_{1}^{h} ; L_{2}\left(\vartheta^{h}\right)\right\|^{-2}\left(h^{2}(h+\Delta h)^{-2}\left\|\partial_{y} w ; L_{2}\left(\vartheta^{h}\right)\right\|^{2}+\left\|\partial_{z} w ; L_{2}\left(\vartheta^{h}\right)\right\|^{2}\right) \\
& \leq\left\|w_{1}^{h} ; L_{2}\left(\vartheta^{h}\right)\right\|^{-2}\left\|\nabla_{x} w_{1}^{h} ; L_{2}\left(\vartheta^{h}\right)\right\|^{2}=\beta_{1}^{h} .
\end{aligned}
$$

We write the one-dimensional Friedrichs inequality

$$
\int_{0}^{a}\left|\frac{d W}{d y}(y)\right|^{2} d y \geq \frac{\pi^{2}}{4 a^{2}} \int_{0}^{a}|W(y)|^{2} d y, \quad W \in C^{1}[0, a], W(0)=0,
$$

we put $a(z)=1+h^{-1} z$ and integrate the result over $z \in(-h, 0)$. Since $\pi^{2} a(z)^{-2} \geq \pi^{2}$, we have

$$
\left\|\nabla_{x} w ; L_{2}\left(\vartheta^{h}\right)\right\|^{2} \geq\left\|\partial_{y} w ; L_{2}\left(\vartheta^{h}\right)\right\|^{2} \geq \frac{\pi^{2}}{4}\left\|w ; L_{2}\left(\vartheta^{h}\right)\right\|^{2}, \quad w \in \stackrel{\circ}{H}^{1}\left(\vartheta^{h} ; \tau^{h}\right),
$$

which yields the left inequality in (2.2).

Let $2 h_{n}$ be the side of the regular $n$-gon circumscribed about the unit circle. We form even extensions of the function $w_{1}^{h_{n}}$ over the hypotenuses and those legs of the triangles $\vartheta^{h}$ and of its copies on which the Neumann conditions are posed, obtaining a positive eigenfunction of the Dirichlet problem in the $n$-gon; we find that $\beta_{1}^{h_{n}}$ is the first eigenvalue of this problem. Consequently, $\beta_{1}^{h_{n}} \rightarrow \beta_{1}^{0}$ as $h \rightarrow \infty$. Since monotonicity is already checked, this makes the right inequality in (2.2) evident. 


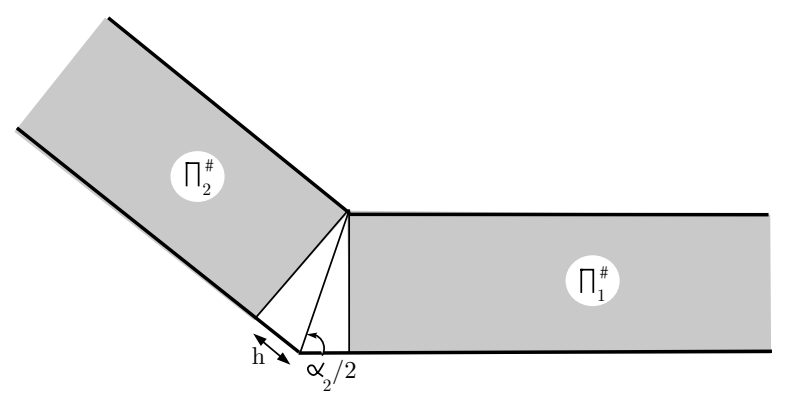

FiguRE 14. Splitting of cranked waveguide.

Theorem 2.2. The operator $A$ of the mixed boundary-value problem in the cranked waveguide $\Omega$ depicted in Figures 14 and 1a with the angle $\alpha_{2} \in(0, \pi)$ measured between rays carrying the Dirichlet conditions, has the spectrum $\left[\pi^{2} / 4, \infty\right)$ which is continuous, and has no discrete spectrum.

Proof. It suffices to check that there are no eigenvalues on $\left(0, \pi^{2} / 4\right)$. Let $u \in \stackrel{\circ}{H}^{1}\left(\Omega ; \Gamma_{D}\right)$ be a solution of problem (1.1), (1.5) for $\lambda<\pi^{2} / 4$. We put $\Pi_{p}^{\#}=\left\{x \in \Pi_{p}: z_{p}>\right.$ $\left.\cot \left(\alpha_{2} / 2\right)\right\}$ (see Figure 14) and observe that, by the Friedrichs inequality on the interval $(0,1)$ (relation (2.4) with $a=1$ ), we have

$$
\left\|\nabla_{x} u ; L_{2}\left(\Pi_{p}^{\#}\right)\right\|^{2} \geq \int_{0}^{1} \int_{\cot \left(\alpha_{2} / 2\right)}^{\infty}\left|\frac{\partial u}{\partial y_{p}}(x)\right|^{2} d y_{p} d z_{p} \geq \frac{\pi^{2}}{4}\left\|u ; L_{2}\left(\Pi_{p}^{\#}\right)\right\|^{2} .
$$

Also, using Lemma 2.1 and, in particular, inequality (2.5), we obtain

$$
\left\|\nabla_{x} u ; L_{2}\left(\Pi_{p} \backslash \Pi_{p}^{\#}\right)\right\|^{2} \geq \beta_{1}^{h}\left\|u ; L_{2}\left(\Pi_{p} \backslash \Pi_{p}^{\#}\right)\right\|^{2} \geq \frac{\pi^{2}}{4}\left\|u ; L_{2}\left(\Pi_{p} \backslash \Pi_{p}^{\#}\right)\right\|^{2} .
$$

Adding the above estimates and recalling identity (1.6), we get

$$
\begin{aligned}
\lambda\left\|u ; L_{2}(\Pi)\right\|^{2} & =\left\|\nabla_{x} u ; L_{2}(\Pi)\right\|^{2} \\
& =\sum_{p=1}^{2}\left(\left\|\nabla_{x} u ; L_{2}\left(\Pi_{p}^{\#}\right)\right\|^{2}+\left\|\nabla_{x} u ; L_{2}\left(\Pi_{p} \backslash \Pi_{p}^{\#}\right)\right\|^{2}\right) \geq \frac{\pi^{2}}{4}\left\|u ; L_{2}(\Pi)\right\|^{2} .
\end{aligned}
$$

It follows that $\left\|u ; L_{2}(\Pi)\right\|=0$, whence $u=0$, so that $\lambda$ is not an eigenvalue.

Remark 2.1. In particular, the proof of Theorem 2.2 implies the last claim in Example1.1.

3. Cranked and broken waveguides with Dirichet conditions. The domain $\Omega$ in Figure 13 is composed of two identical slanted half-strips $\Pi_{1}$ and $\Pi_{2}$ (Figure 11a or 11b). The calculations made in Example 1.2 for the case where $k_{0}=k_{1}$ (Figure 12c) show that the function (2.1) defined in accordance with the lower lines in (1.8) and (1.11), satisfies

$$
\left\|\nabla_{x} u^{\varepsilon} ; L_{2}(\Omega)\right\|^{2}-\pi^{2}\left\|u^{\varepsilon} ; L_{2}(\Omega)\right\|^{2}=O(\varepsilon) .
$$

Using this relation, we repeat the calculations used in the proof of Theorem 1.1(2), with minor changes caused by the presence of two exits to infinity. Thus, the inequality

$$
\left\|u^{\varepsilon}+\sqrt{\varepsilon} v ; L_{2}(\Omega)\right\| \leq \pi^{-2}\left\|\nabla_{x} u^{\varepsilon}+\sqrt{\varepsilon} \nabla_{x} v ; L_{2}(\Omega)\right\|,
$$


implied by the assumption $\underline{\sigma}=\lambda_{\dagger}=\pi^{2}$ (cf. the previous formula (1.24)), reshapes to the form

$$
\begin{aligned}
C \varepsilon & \geq 2 \sqrt{\varepsilon} \sum_{p=1}^{2} \int_{\Pi_{p}}\left(\frac{\partial U_{\dagger}}{\partial y_{p}}\left(y_{p}\right) \frac{\partial v}{\partial y_{p}}(x)-\pi^{2} U_{\dagger}\left(y_{p}\right) v(x)\right) d x+O(\varepsilon) \\
& =2 \sqrt{\varepsilon} \sum_{p=1}^{2} \int_{B_{p}} v(x) \cos \beta_{p} \frac{\partial U_{\dagger}}{\partial y_{p}}\left(y_{p}\right) d s_{x}+O(\varepsilon) .
\end{aligned}
$$

Here $v$ is an arbitrary smooth function with compact support in $\Omega$; integration by parts gave rise to the factor $\cos \beta_{p}$, where $\beta_{p}=\beta \in(0, \pi / 2)$ is the angle between the axis $y_{p}$ and the normal to the end $B_{p}$ of the half-strip $\Pi_{p}$. Observe that $\left(\partial U_{\dagger} / \partial y\right)(y)=\pi \cos (\pi y)$ and $d s_{x}=(\sin \beta)^{-1} d y$. Consequently, the right-hand side of (2.10) is equal to

$$
4 \sqrt{\varepsilon} \cot \beta \int_{0}^{1} \cos (\pi y) v(y, y \cot \beta) d y+O(\varepsilon) .
$$

Now it becomes clear that an appropriate choice of the test function $v$ makes the integral in (2.11) positive, so that inequality (2.10) fails. This contradiction shows that $\underline{\sigma}<\pi^{2}$, whence we see that there exists a point $\lambda=\underline{\sigma} \in\left(0, \pi^{2}\right)$ belonging to the discrete spectrum.

Theorem 2.3. The discrete spectrum of the operator of the Dirichlet problem (1.1), (1.4) in a cranked waveguide $\Omega$ as in Figure 1 is nonempty for any angle $\alpha_{2} \neq \pi$ between the axes of the half-strips that form this waveguide.

The approach described above works also in the case of broken waveguides as in Figure 5. Then the test function $u^{\varepsilon}$ involves the factor $\sin \left(\pi y_{p}\right)$ in the slanted half-strips $\Pi_{1}$ and $\Pi_{2}$, as well as in the trapezoid $\Pi_{3}$ of unit height that connects $\Pi_{1}$ and $\Pi_{2}\left(\Pi_{3}\right.$ can degenerate to a triangle). As another series of examples, we mention several parallel waveguides as in (1.9) connected by bridges (Figure 6). The test function in the pointed half-strips (see Figure 6) is given by the same formula as before, and in the hexagon (or rhombus in the degenerate case) it is equal to $\cos (\pi z)$ (the origin of the $z$-axis is marked with a bold point). Of course, the number of the waveguides and bridges may be arbitrary.

4. Smoothly bent waveguides. Let $L$ be a smooth arc on the plane, coinciding with straight rays $L_{ \pm}$outside the disk $\mathbb{B}_{R}$ of radius $R$. Let $\Omega$ be given by (1.2); we assume that this domain $\Omega$ is a curvilinear strip of unit width (see Figure 7, a and b), i.e.,

$$
\Omega=\{x: s \in \mathbb{R},-1 / 2<n<1 / 2\} ;
$$

here $(n, s)$ is the coordinate system near $L ; s$ is the arclength on $L$, and $n$ is the oriented distance to $L$. The above assumption excludes, in particular, all self-intersecting curves (Figure 7c).

In the curvilinear coordinates, the Laplace operator takes the form

$$
\Delta_{x}=\frac{1}{1+n \varkappa(s)} \frac{\partial}{\partial n}(1+n \varkappa(s)) \frac{\partial}{\partial n}+\frac{1}{1+n \varkappa(s)} \frac{\partial}{\partial s} \frac{1}{1+n \varkappa(s)} \frac{\partial}{\partial s},
$$

and the Dirichlet integral looks like this:

$$
D(u ; \Omega)=\int_{\mathbb{R}} \int_{-1 / 2}^{1 / 2}\left(\left|\frac{\partial u}{\partial n}(n, s)\right|^{2}+\frac{1}{(1+n \varkappa(s))^{2}}\left|\frac{\partial u}{\partial s}(n, s)\right|^{2}\right)(1+n \varkappa(s)) d n d s .
$$

Here $\varkappa(s)$ is the curvature of the $\operatorname{arc} L$ at $s$. Observe that, first, we do not distinguish notationally a point $s$ and its coordinate on $L$, and second, we preserve the symbol of a function after rewriting it in the coordinates $n$ and $s$. 
We consider the test function

$$
u^{\varepsilon}(x)=\exp (-\varepsilon|s|) \cos (\pi n)
$$

similar to (1.20). We have

$$
\begin{aligned}
\left\|u^{\varepsilon} ; L_{2}(\Omega)\right\|^{2} & =\int_{\mathbb{R}} \int_{-1 / 2}^{1 / 2}\left|u^{\varepsilon}(n, s)\right|^{2}(1+n \varkappa(s)) d n d s \\
& =\int_{\mathbb{R}} \exp (-2 \varepsilon|s|)\left(\int_{-1 / 2}^{1 / 2}|\cos (\pi n)|^{2}(1+n \varkappa(s)) d n\right) d s \\
& =\frac{1}{2} \int_{\mathbb{R}} \exp (-2 \varepsilon|s|) d s \\
& =\frac{1}{2 \varepsilon}+O(\varepsilon) .
\end{aligned}
$$

Since the expression $|\cos (\pi n)|^{2} n \varkappa(s)$ is even relative to the variable $n \in(-1 / 2,1 / 2)$, the integral (2.16) is independent of the curvature $\varkappa$. Similarly, for the Dirichlet integral (2.14) we obtain

$$
D\left(u^{\varepsilon} ; \Omega\right)=\frac{\pi^{2}}{2 \varepsilon}+O(\varepsilon)
$$

the contribution of the expression

$$
(1+n \varkappa(s))^{-1}\left|\partial_{s} u^{\varepsilon}(n, s)\right|^{2}
$$

being $O(\varepsilon)$ due to a small factor in the exponent on the right in (2.15). So, by (2.16) and (2.17), the test function (2.15) satisfies relation (2.8), which allows us to apply the trick used in the proof of Theorem 1.1(2). Namely, for the new test function $u^{\varepsilon}+\sqrt{\varepsilon} v$ we have

$$
\begin{aligned}
\pi^{2}\left\|u^{\varepsilon}+\sqrt{\varepsilon} v ; L_{2}(\Omega)\right\| & =\frac{\pi^{2}}{2 \varepsilon}+2 \sqrt{\varepsilon} \pi^{2} \int_{\Omega} v(x) \cos (\pi n) d x+O(\varepsilon), \\
D\left(u^{\varepsilon}+\sqrt{\varepsilon} v ; \Omega\right) & =\frac{\pi^{2}}{2 \varepsilon}+2 \sqrt{\varepsilon} \int_{\Omega} \nabla_{x} v(x) \cdot \nabla_{x} \cos (\pi n) d x+O(\varepsilon) .
\end{aligned}
$$

The assumption (1.24) about the absence of the discrete spectrum implies inequality (2.9); using (2.18) and the Green formula, we can transform (2.9) as follows:

$$
\begin{aligned}
C \varepsilon & \geq 2 \sqrt{\varepsilon} \int_{\Omega}\left(\pi^{2} v(x) \cos (\pi n)-\nabla_{x} v(x) \cdot \nabla_{x} \cos (\pi n)\right) d x \\
& =-2 \sqrt{\varepsilon} \int_{\Omega} v(x)\left(\Delta_{x}-\pi^{2}\right) \cos (\pi n) d x .
\end{aligned}
$$

An appropriate choice of a smooth function $v$ with small compact support leads to the desired contradiction, showing that the discrete spectrum $\sigma_{d}$ is nonempty provided that

$$
\left(\Delta_{x}-\pi^{2}\right) \cos (\pi n) \neq 0 \text { for some point } x^{0} \in \Omega .
$$

By (2.13), the expression on the left equals

$$
-\pi \varkappa(s)(1+n \varkappa(s))^{-1} \sin (\pi n)
$$

and the required point $x^{0}$ can be found easily if the curvature is nonzero somewhere on $L$.

Theorem 2.4 (see [1]). If the smooth arc $L$ is not a straight line, then the discrete spectrum of the operator of the Dirichlet problem in the domain (2.12) is nonempty. 
The facts mentioned immediately after Theorem 2.3 can be interpreted as a generalization to the case where $L$ is a broken line. The inequalities $-1 / 2<n<1 / 2$ in the definition (2.12) of the domain $\Omega$ can be replaced with the inequalities $h-1 / 2<n<h+1 / 2$ with any $h \in \mathbb{R}$, because

$$
\int_{h-1 / 2}^{h+1 / 2} n\left((\cos (\pi n))^{2}-(\sin (\pi n))^{2}\right) d n=0,
$$

and all the above calculations and arguments remain valid. The existence of an eigenvalue $\lambda \in\left(0, \pi^{2}\right)$ for the waveguide depicted in Figure 7 c (a self-intersecting curve $L$ ) will be established in Subsection 5 of $\S 2$.

5. Branching waveguides with Dirichlet conditions. Consider the Dirichlet problem (1.1), (1.4) for $N>2$ (Figures $2 \mathrm{a}$ and 3a). We split the domain $\Omega$ into half-strips $\Pi_{1}, \ldots, \Pi_{N}$ with pointed ends, connecting by segments the origin $\mathcal{O}$ and the points $P^{1}, \ldots, P^{N}$ of intersection of the lateral sides of half-strips. In the local coordinates $\left(y_{p}, z_{p}\right)$, each set $\Pi_{p}$ takes the form (1.12) with the profile function (1.28), and $k_{0}^{p}>0$. Since $N \geq 3$, the angle $\beta_{p}$ at the vertex of the pointed end is strictly less than $\pi$, i.e., it is obtuse, right-angled, or acute. Thus, $k_{1}^{p}<k_{0}^{p}$ (recall that the end of a half-strip is defined by the equation $z_{p}=-H_{p}\left(y_{p}\right)$ ), and the integral (1.29) is negative for each of the half-strips $\Pi_{p}$. It remains to observe that the function $u^{\varepsilon}$ given by the formula

$$
u^{\varepsilon}(x)= \begin{cases}\sin \left(\pi y_{p}\right) & \text { if } x \in \Pi_{p} \backslash \Pi_{p}^{\#}, \\ \sin \left(\pi y_{p}\right) \exp \left(-\varepsilon z_{p}\right) & \text { if } x \in \Pi_{p}^{\#}\end{cases}
$$

is continuous and piecewise smooth in $\Omega$, because $\sin \left(\pi y_{p}\right)=\sin \left(\pi y_{q}\right)$ on the common segment $B_{p q}$ of the pointed ends of two neighboring half-strips $\Pi_{p}$ and $\Pi_{q}$. The origin of the local coordinates $\left(y_{p}, z_{p}\right)$ is fixed in such a way that $\Pi_{p}^{\#}=\left\{x: y_{p} \in(0,1), z_{p}>0\right\}$ is the largest half-strip with right-angled end contained in $\Pi_{p}$. As a result, we arrive at the relation

$$
\begin{aligned}
\underline{\sigma} & \leq \frac{\left\|\nabla_{x} u^{\varepsilon} ; L_{2}(\Omega)\right\|^{2}}{\left\|u^{\varepsilon} ; L_{2}(\Omega)\right\|^{2}}=\frac{\sum_{p=1}^{N}\left\|\nabla_{x} u^{\varepsilon} ; L_{2}\left(\Pi_{p}^{\#}\right)\right\|^{2}+\sum_{p=1}^{N}\left\|\nabla_{x} u^{\varepsilon} ; L_{2}\left(\Pi_{p} \backslash \Pi_{p}^{\#}\right)\right\|^{2}}{\sum_{p=1}^{N}\left\|u^{\varepsilon} ; L_{2}\left(\Pi_{p}^{\#}\right)\right\|^{2}+\sum_{p=1}^{N}\left\|u^{\varepsilon} ; L_{2}\left(\Pi_{p} \backslash \Pi_{p}^{\#}\right)\right\|^{2}} \\
& =\frac{\sum_{p=1}^{N} \int_{0}^{1} \int_{0}^{\infty} e^{-2 \varepsilon z_{p}}\left(\pi^{2}\left(\cos \left(\pi y_{p}\right)\right)^{2}+\varepsilon^{2}\left(\sin \left(\pi y_{p}\right)\right)^{2}\right) d z_{p} d y_{p}+\pi^{2} \sum_{p=1}^{N} \int\left(\cos \left(\pi y_{p}\right)\right)^{2} d x}{\sum_{p=1}^{N} \int_{0}^{1} \int_{0}^{\infty} e^{-2 \varepsilon z_{p}}\left(\sin \left(\pi y_{p}\right)\right)^{2} d z_{p} d y_{p}+\sum_{p=1}^{N} \int_{\Pi_{p} \backslash \Pi_{p}^{\#}}\left(\sin \left(\pi y_{p}\right)\right)^{2} d x} \\
& \leq \frac{\frac{N}{2 \varepsilon} \pi^{2}+c \varepsilon+\pi^{2} \sum_{p=1}^{N} \int_{\Pi_{p} \backslash \Pi_{p}^{\#}}\left(\cos \left(\pi y_{p}\right)\right)^{2} d x}{\frac{N}{2 \varepsilon}+\sum_{p=1}^{N} \int_{\Pi_{p} \backslash \Pi_{p}^{\#}}\left(\sin \left(\pi y_{p}\right)\right)^{2} d x} \leq \pi^{2}\left(1+\frac{2}{N} \varepsilon \sum_{p=1}^{N} I_{p}+O\left(\varepsilon^{2}\right)\right),
\end{aligned}
$$

where

$$
\begin{aligned}
I_{p} & \left.=\int_{\Pi_{p} \backslash \Pi_{p}^{\#}}\left(\cos \left(\pi y_{p}\right)\right)^{2}-\left(\sin \left(\pi y_{p}\right)\right)^{2}\right) d x+\sum_{p=1}^{N} \int_{\Pi_{p} \backslash \Pi_{p}^{\#}}\left(\sin \left(\pi y_{p}\right)\right)^{2} d x \\
& \leq \int_{0}^{1} \int_{-H^{p}\left(y_{p}\right)}^{0}\left(\cos \left(2 \pi y_{p}\right)\right)^{2} d z_{p} d y_{p}=-\int_{0}^{1} H^{p}\left(y_{p}\right)\left(\cos \left(2 \pi y_{p}\right)\right)^{2} d y_{p}<0,
\end{aligned}
$$

and the $H^{p}$ are the convex $\left(k_{1}^{p}<k_{0}^{p}\right)$ piecewise linear profile functions (1.28) that describe the ends of the pointed half-strips $\Pi_{p}$ forming the branching waveguide $\Omega$. Using (2.20), we choose $\varepsilon>0$ so small that relation (2.19) takes the form $\underline{\sigma}<\pi^{2}$, thus establishing the next claim. 

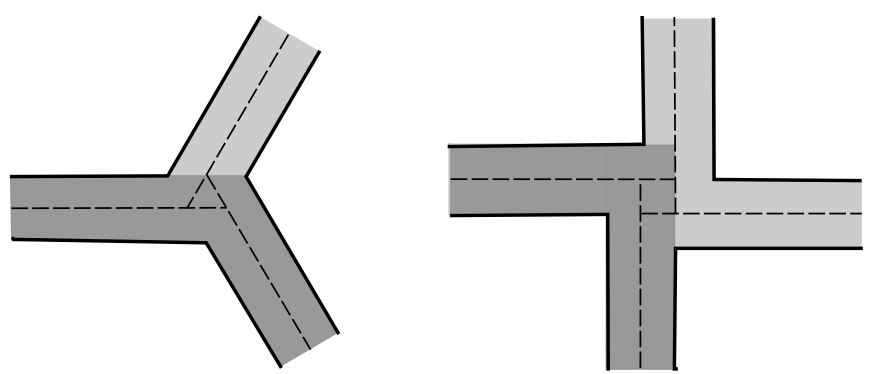

FiguRE 15. Noncentered branching waveguides.

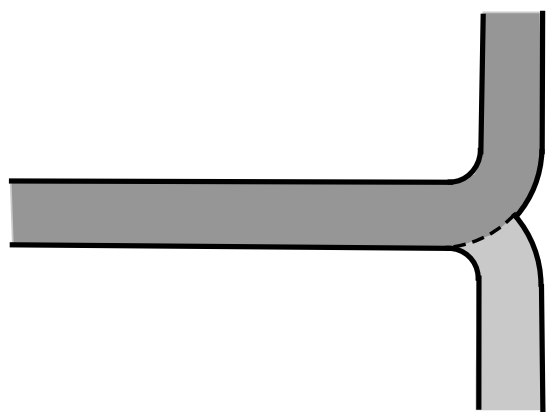

FiguRE 16. A branching curved waveguide.

Theorem 2.5. The operator $A$ of the Dirichlet problem (1.1), (1.4) in a domain $\Omega$ (branching waveguide) with $N>2$ exist to infinity, as depicted in Figures 2a and 3a in the cases where $N=3$ and $N=4$, respectively, always has an eigenvalue on the interval $\left(0, \pi^{2}\right)$, i.e., the discrete spectrum of $A$ is nonempty.

Remark 2.2. Theorem 2.5 can be verified with the help of formula (1.19). Indeed, by what is proved in Theorem 2.3 , any of the cranked waveguides $\Omega^{<}$obtained by cutting off the "excess" exits to infinity from the branching waveguide $\Omega$, has an eigenvalue $\lambda^{<}<\pi^{2}$ with an eigenfunction $u^{<} \in \stackrel{\circ}{H}^{1}\left(\Omega^{<}\right)$. Observing that the Dirichlet problems in $\Omega$ and $\Omega^{<}$ have identical continuous spectrum $\sigma_{c}=\sigma_{c}^{<}=\left[\pi^{2},+\infty\right)$, we substitute the eigenfunction $u^{<}$extended by zero outside of $\Omega^{<}$in formula (1.19):

$$
\underline{\sigma}=\inf _{u \in \dot{H}^{1}(\Omega) \backslash\{0\}} \frac{\left\|\nabla_{x} u ; L_{2}(\Omega)\right\|^{2}}{\left\|u ; L_{2}(\Omega)\right\|^{2}} \leq \frac{\left\|\nabla_{x} u^{<} ; L_{2}\left(\Omega^{<}\right)\right\|^{2}}{\left\|u^{\angle} ; L_{2}\left(\Omega^{<}\right)\right\|^{2}}=\lambda^{<}<\pi^{2} .
$$

As usual, this implies that $\sigma_{d} \neq \varnothing$.

It should be emphasized that the simple approach described in Remark 2.2 may fail to work in the case of waveguides with piecewise constant width (see Example 2.1). On the other hand, the waveguides depicted in Figures 2 and 3a are centered, i.e., the midlines of the strips forming them intersect at one point, but for decentered waveguides (see Figure 15) the variational method that has led us to Theorem [2.5] is no longer applicable, yet the simple considerations of Remark 2.2 (in particular, formula (2.21)) work well (the cranked waveguides whose eigenvalues majorize those of the corresponding branching waveguides are deeply tinted in Figure 15).

The smoothly bent waveguides can be treated as described in Remark 2.2 for the case of branching ones (see Figure 16). Into the self-intersecting waveguide as in Figure 7, we 


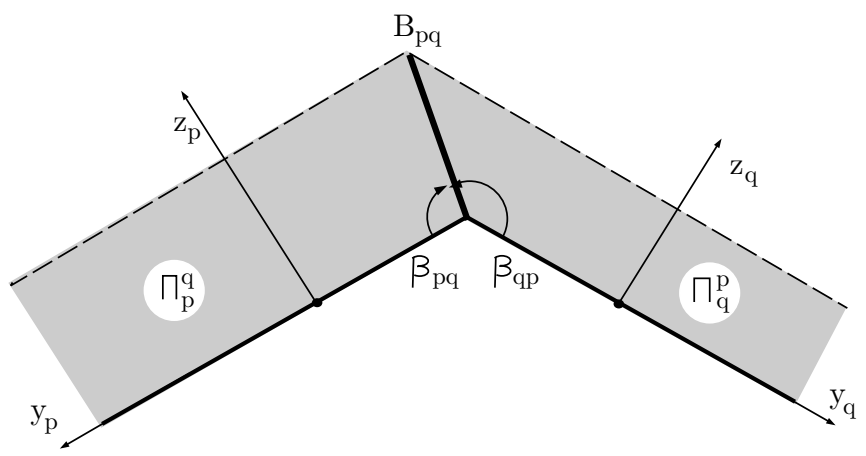

FiguRE 17. Part of a waveguide with piecewise constant width.

can place a cranked waveguide with $\alpha_{2}=\pi / 2$ (the deeply tinted fragment of the figure); hence, the discrete spectrum is still nonempty in this case.

6. Branching waveguides with piecewise constant width. Let $\Pi_{1}^{\infty}, \ldots, \Pi_{N}^{\infty}$ be strips of width $l_{1}, \ldots, l_{N}, N \geq 3$, and let the following be true for some natural $m \in$ $(0, N)$ :

$$
1=l_{1}=\cdots=l_{m}>l_{m+1} \geq \cdots \geq l_{N} .
$$

Suppose that the midlines of the strips intersect at the point $\mathcal{O}$. From the corresponding pointed half-strips, we build a branching waveguide (see Figure 2a). Namely, from the point $P^{p q}$ of intersection of sides of two neighboring half-strips with right-angled ends, we draw the segment $B^{p q}$ to the point $\mathcal{O}$ (Figure 17). This segment is part of the pointed end of half-strips $\Pi_{p}$ and $\Pi_{q}$, which are components of the waveguide $\Omega$; such a construction should be done for all pairs of neighboring half-strips.

Like in the preceding subsection, we consider the Dirichlet problem (1.1), (1.4) and define a test function $u^{\varepsilon}$ in the half-strips $\Pi_{p}$ of unit width, $p=1, \ldots, m$, by the formulas

$$
u^{\varepsilon}(x)= \begin{cases}\sin \left(\pi y_{p}\right) & \text { if } x \in \Pi_{p}, z_{p}<0 \\ \exp \left(-\varepsilon z_{p}\right) \sin \left(\pi y_{p}\right) & \text { if } x \in \Pi_{p}, z_{p}>0 .\end{cases}
$$

The origin of the local Cartesian coordinate system $\left(y_{p}, z_{p}\right)$ is assumed to lie at a sufficiently large distance $R$ from the point $\mathcal{O}$ (Figure 17), in order that, in the junction zone itself, the function $u^{\varepsilon}$ coincide with sine functions without exponential factors. For $q=m+1, \ldots, N$, in the half-strip $\Pi_{q}$ of width $l_{q}<1$ (see (2.22) $)$, the test function has the form

$$
u^{\varepsilon}(x)= \begin{cases}\sin \left(l_{q}^{-1} \pi y_{q}\right) & \text { if } x \in \Pi_{q}, z_{q}<0, \\ \exp \left(-\pi\left(l_{q}^{-2}-1\right)^{1 / 2} z_{q}\right) \sin \left(l_{q}^{-1} \pi y_{q}\right) & \text { if } x \in \Pi_{q}, z_{q}>0 .\end{cases}
$$

The function written in the lower line in 2.23) decays exponentially at infinity and satisfies the Helmholtz equation (1.1) with the parameter $\lambda=\pi^{2}$ in the entire strip $\Pi_{q}^{\infty}$. It is important that the traces of $u^{\varepsilon}$ on $\overline{B^{p q}}=\partial \Pi_{p} \cap \partial \Pi_{q}$ calculated from the sides of $\Pi_{p}$ and $\Pi_{q}$ coincide. This is obvious, because the level lines of the functions $\sin \left(l_{p}^{-1} \pi y_{p}\right)$ and $\sin \left(l_{q}^{-1} \pi y_{q}\right)$ intersect $B_{p q}$ at a common point; however this fact is crucial, allowing us to conclude that $u^{\varepsilon} \in \stackrel{\circ}{H}^{1}(\Omega)$.

For each half-strip $\Pi_{p}$, its contribution to the continuous spectrum $\sigma_{c}$ of $\Omega$ is equal to $\left[\pi^{2} l_{p}^{-2},+\infty\right)$, and the entire $\sigma_{c}$ is determined by the half-strip of maximal width. 
Recalling (2.22), we see that $\sigma_{c}=\left[\pi^{2},+\infty\right)$. If $\underline{\sigma}=\pi^{2}$, then, by (1.19),

$$
I(\Omega):=\int_{\Omega}\left(\pi^{2}\left|u^{\varepsilon}(x)\right|^{2}-\left|\nabla_{x} u^{\varepsilon}(x)\right|^{2}\right) d x \leq 0 .
$$

We compute the above integral over the half $\Pi_{p}^{q}$ of the half-strip that adjoints the segment $B_{p q}$ (Figure 17). For $p=1, \ldots, m$ we have

$$
\begin{aligned}
I\left(\Pi_{p}^{q}\right)= & \int_{0}^{1 / 2} \int_{0}^{\infty}\left(\pi^{2}\left(\sin \left(\pi y_{p}\right)\right)^{2}-\pi^{2}\left(\cos \left(\pi y_{p}\right)\right)^{2}-\varepsilon^{2}\left(\sin \left(\pi y_{p}\right)\right)^{2}\right) \exp \left(-2 \pi \varepsilon z_{p}\right) d z_{p} d y_{p} \\
& \quad+\pi^{2} \int_{0}^{1 / 2} \int_{-R+y_{p} \cot \beta_{p q}}^{0}\left(\left(\sin \left(\pi y_{p}\right)\right)^{2}-\left(\cos \left(\pi y_{p}\right)\right)^{2}\right) d z_{p} d y_{p} \\
= & O(\varepsilon)-\pi^{2} \int_{0}^{1 / 2}\left(-R+y_{p} \cot \beta_{p q}\right) \cos \left(2 \pi y_{p}\right) d y_{p} \\
= & \frac{1}{2} \cot \beta_{p q}+O(\varepsilon) .
\end{aligned}
$$

Here $\beta_{p q} \in(0, \pi)$ is the angle between the axis of $\Pi_{p}$ and the segment $B_{p q}$.

If $q>m$ and $l_{q}<1$, then

$$
\begin{aligned}
I\left(\Pi_{q}^{p}\right)= & \pi^{2} \int_{0}^{l_{q} / 2} \int_{0}^{\infty}\left(\left(\sin \left(l_{q}^{-1} \pi y_{q}\right)\right)^{2}-l_{q}^{-2}\left(\begin{array}{c}
\left.\left.\cos \left(l_{q}^{-1} \pi y_{q}\right)\right)^{2}-\left(l_{q}^{-2}-1\right)\left(\sin \left(l_{q}^{-1} \pi y_{q}\right)\right)^{2}\right) \\
\times \exp \left(-2 \pi\left(l_{q}^{-2}-1\right)^{1 / 2} z_{q}\right) d z_{q} d y_{q}
\end{array}\right.\right. \\
& \quad+\pi^{2} \int_{0}^{l_{q} / 2} \int_{-R+y_{q} \cot \beta_{q p}}^{0}\left(\left(\sin \left(l_{q}^{-1} \pi y_{q}\right)\right)^{2}-l_{q}^{-2}\left(\cos \left(\pi y_{q}\right)\right)^{2}\right) d z_{q} d y_{q} \\
= & \frac{\pi}{4}\left(1-l_{q}^{2}\right)^{1 / 2}+\frac{\pi^{2}}{4} R\left(1-l_{q}^{2}\right)-\frac{\pi}{16}\left(1-l_{q}^{2}\right) \cot \beta_{p q}+\frac{1}{4}\left(1+l_{q}^{2}\right) \cot \beta_{p q} \\
= & \frac{1}{2} \cot \beta_{p q}+\left(1-l_{q}^{2}\right)^{1 / 2} J_{q p}\left(l_{q}\right) .
\end{aligned}
$$

Here $J_{q p}$ is a continuous function. Finally, we get the formulas

$$
\begin{aligned}
& I(\Omega)=I^{\prime}(\Omega)+\sum_{p q}\left(1-l_{q}^{2}\right)^{1 / 2} J_{q p}\left(l_{q}\right)+O(\varepsilon), \\
& I^{\prime}(\Omega)=\frac{1}{2} \sum_{p q}\left(\cot \beta_{p q}+\cot \beta_{q p}\right),
\end{aligned}
$$

where on the right-hand side of (2.26), summation is over all intersection points $P^{p q}$ for the lateral sides of neighboring half-strips, and on the right in (2.25), summation is over all $P^{p q}$ such that $l_{q}<1$.

Repeating the calculations in Example 1.2 and the arguments before Theorem 2.5, we see that the sum (2.26) is negative. Thus, if the differences $1-l_{q}, q=m+1, \ldots, N$, are small, then the parameter $\varepsilon>0$ can be chosen so that the expression (2.25) be negative. Now, for the same reasons as before, the following statement is true.

Theorem 2.6. For any branching waveguide $\Omega$ composed of half-strips $\Pi_{p}$ of width $l_{p}$, $p=1, \ldots, N$, and satisfying (2.22), there exists $\delta>0$ such that if

$$
l_{q} \in(1-\delta, 1), \quad q=m+1, \ldots, N,
$$

then the Dirichlet problem (1.1), (1.4) has an eigenvalue $\lambda \in\left(0, \pi^{2}\right)$, i.e., the discrete spectrum of the corresponding operators $A$ is nonempty. 
Remark 2.3. 1) For the Dirichlet problem, the method of the proof of Theorem 2.6 does not work in the case of cranked waveguides with piecewise-constant width (Figure 8c), because the calculations in Example 1.2 (the case where $k_{0}=k_{1} \neq 0$; see Figure 12c) show that the sum (2.26) vanishes. Therefore, the simple trick described in Remark 2.2 allows us to find a point of the discrete spectrum for the $T$-like waveguide $\Omega^{h}$ (Figure $8 \mathrm{~b}$ ) only if the width $h$ of the vertical half-strip is equal to 1 : whenever $h>1$, the continuous spectrum $\sigma_{c}$ extends up to the ray $\left[h^{-2} \pi^{2}, \infty\right)$, but if $h<1$, so that $\sigma_{c}=\left[\pi^{2}, \infty\right)$, then no cranked waveguide of unit width fits into $\Omega^{h}$.

2) In the proof of Theorem 2.1, the cranked waveguide in Figure 1b (with mixed boundary conditions) was split into two slanted half-strips, each satisfying the strict inequality (1.16). Therefore, in the same way we can check the existence of an eigenvalue below the continuous spectrum threshold for a cranked waveguide of the same type with one of the sleeves slightly varied.

Example 2.1. Consider the $T$-like junction of a strip and a half-strip of width 1 and $h$, respectively (see Figure 8b, and Remark 2.3). Theorems 2.5 and 2.6 indicate points of discrete spectrum for $h \in\left(1-h_{-}, 1+h_{+}\right)$; here the $h_{ \pm}$are some positive quantities (note that, for $h>1$, to apply Theorem 2.6 directly, we must perform the coordinate contraction $x \mapsto h^{-1} x$; see the requirement (2.22) $)$. In Remark 2.3 it was already mentioned that no other conclusions are possible on the basis of the results obtained. Nevertheless, the conjecture that the discrete spectrum is nonempty for any $h \in(0,1)$ is fairly plausible. By Remark 2.2. to check this conjecture it suffices to explore the $T$-like waveguide in the limit as $h \rightarrow+0$. Apparently, the discrete spectrum is empty for $h$ sufficiently large. We verify that for $h \leq 1$ the discrete spectrum can consist of at most one point. Let $\sigma_{d} \neq \varnothing$ and let $\lambda_{1}<\pi^{2}$ be the smallest eigenvalue. By the maximum principle, the corresponding eigenfunction $u_{1}$, which decays exponentially as $|x| \rightarrow+\infty$, can be assumed to be positive in $\Omega^{h}$. We invoke the maximum principle (see [19, Theorem 10.2.2]):

$$
\lambda_{j}=\max _{\mathcal{E}_{j}} \inf _{v \in \mathcal{E}_{j} \backslash\{0\}} \frac{\left\|\nabla_{x} v ; L_{2}(\Omega)\right\|^{2}}{\left\|v ; L_{2}(\Omega)\right\|^{2}},
$$

where $\mathcal{E}_{j}$ is an arbitrary subspace in $\stackrel{\circ}{H}^{1}(\Omega)$ of codimension $j-1$; in particular, $\mathcal{E}_{1}=$ $\stackrel{\circ}{H}^{1}(\Omega)$. For $j=2$, for the role of the subspace we take

$$
\mathcal{E}_{2}=\left\{v \in \stackrel{\circ}{H}^{1}(\Omega): \int_{Q^{h}} v(x) \sin \left(\pi \frac{z}{2}\right) d y d z=0\right\} .
$$

Here $Q^{h}$ is the rectangle $\{x=(y, z): y \in(0, h), z \in(0,1)\}$ (the coordinate axes are indicated in Figure 8b, and the rectangle itself is deeply tinted). The codimension of the subspace (2.28) is one, because $v$ is subject to one linear restriction. If the discrete spectrum has an eigenvalue $\lambda_{2}$ then, by (2.27), we have

$$
\lambda_{2} \geq \inf _{v \in \mathcal{E}_{2} \backslash\{0\}} \frac{\left\|\nabla_{x} v ; L_{2}(\Omega)\right\|^{2}}{\left\|v ; L_{2}(\Omega)\right\|^{2}} .
$$

The first two eigenvalues of the mixed boundary-value problem in $Q^{h}$ (the Dirichlet conditions are imposed on the lower base $q^{h}$ of $Q^{h}$ ) are equal to $\mu_{1}=\pi^{2} / 4$ and $\mu_{2}=\min \left\{\pi^{2}, h^{-2} \pi^{2}+\pi^{2} / 4\right\}=\pi^{2}$, and the first eigenfunction takes the form $\sin (\pi z / 2)$. Consequently, we can repeat the arguments from the proof of Theorem 2.2 to conclude that, on the subspace (2.28), we have $\left\|v ; L_{2}(\Omega)\right\|^{2} \leq C\left\|\nabla_{x} v ; L_{2}(\Omega)\right\|^{2}$ with $C=$ $\min \left\{\pi^{2}, h^{-2} \pi^{2}, \pi^{2}\right\}$. The first two numbers $\pi^{2}$ and $h^{-2} \pi^{2}$ arose when the Friedrichs inequality was applied on cross sections of half-strips of width $\pi$ and $h<1$ (see (2.6)), and the last copy of $\pi^{2}$ is due to an application of the Poincaré-Friedrichs inequality similar 
to (2.7):

$$
\begin{aligned}
& \left\|\nabla_{x} v ; L_{2}\left(Q^{h}\right)\right\|^{2} \geq \mu_{2}\left\|v ; L_{2}(\Omega)\right\|^{2}, \quad v \in \stackrel{\circ}{H}^{1}\left(Q^{h} ; q^{h}\right), \\
& \int_{Q^{h}} v(x) \sin \left(\pi \frac{z}{2}\right) d y d z=0 .
\end{aligned}
$$

Thus, the left-hand side of (2.29) cannot be less than $\pi^{2}$, so that it cannot happen that $\lambda_{2} \in\left(0, \pi^{2}\right)$.

If $h=2$, then we can use the trick [7] of posing an artificial Dirichlet condition on the symmetry line $\{(y, z): y=0\}$ and the result of Theorem 2.3 about the cranked waveguide $\Omega_{\natural}$ with unit width and right angle, showing that the Dirichlet problems in $\Omega_{\natural}$ and $\Omega^{2}$ have an eigenvalue $\lambda_{\natural} \in\left(0, \pi^{2}\right)$. However, the continuous spectrum of the waveguide $\Omega^{2}$ occupies the ray $\left[\pi^{2} / 4, \infty\right)$, and it is not clear whether the point $\lambda_{\natural}$ falls into the interval $\left(0, \pi^{2} / 4\right)$. If $\lambda_{\natural} \in\left(0, \pi^{2} / 4\right)$, then the discrete spectrum of $\Omega^{2}$ includes at least one eigenvalue less than $\lambda_{\natural}$. Indeed, in accordance with the method of [7, an eigenfunction $u \in \stackrel{\circ}{H}^{1}\left(\Omega^{2}\right)$ is obtained by the odd (relative to $y$ ) extension of a positive

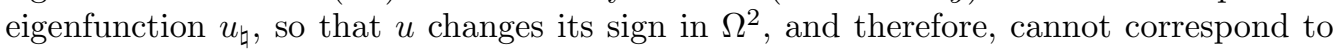
the first eigenvalue of the Dirichlet problem in $\Omega^{2}$.

\section{§3. Many-Dimensional WaVEguides}

1. Semicylinder with curved end. Let $\omega$ be a domain in the Euclidean space $\mathbb{R}^{n-1}$, with Lipschitz boundary $\partial \omega$ and compact closure $\bar{\omega}=\omega \cup \partial \omega$. We split the surface into two parts $\gamma_{D} \neq \varnothing$ and $\gamma_{N}$. We put $\Pi=\omega \times \mathbb{R}$ and, similarly, define the parts $\Gamma_{D}=\gamma_{D} \times \mathbb{R}$ and $\Gamma_{N}=\gamma_{N} \times \mathbb{R}$ of the cylindrical surface $\partial \Pi$. Also, let $H$ be a Lipschitz function on $\bar{\omega}$, let

$$
\Pi^{H}=\left\{x=(y, z): y=\left(y_{1}, \ldots, y_{n-1}\right) \in \omega,-H(y)<z\right\},
$$

and let $\Gamma_{D}^{H}$ and $\Gamma_{N}^{H}$ have similar meaning. In the semicylinder (3.1) with the curved end $\omega^{H}=\{(y, z): y \in \omega, z=-H(y)\}$, we consider the boundary-value problem (1.13), in which $\varpi_{D}^{H}=\Gamma_{D}^{H} \neq \varnothing$ and $\varpi_{N}^{H}=\Gamma_{N}^{H} \cup \omega^{H}$. The variational setting of this problem

$$
\left(\nabla v_{x}, \nabla w_{x}\right)_{\Pi^{H}}=\lambda(v, w)_{\Pi^{H}}, \quad w \in \stackrel{\circ}{H}^{1}\left(\Pi^{h} ; \Gamma_{D}^{H}\right),
$$

gives rise [19, Chapter 10] to an unbounded positive selfadjoints operator $A$ in the space $L_{2}\left(\Pi^{H}\right)$. The continuous spectrum $\sigma_{c}^{H}$ of $A$ coincides with the ray $\left[\lambda_{\dagger}, \infty\right)$, where $\lambda_{\dagger}=\Lambda_{1}$ is the first (simple and positive) eigenvalue of the problem

$$
\left(\nabla_{y} U, \nabla_{y} W\right)_{\omega}=\Lambda(U, W)_{\omega}, \quad W \in \stackrel{\circ}{H}^{1}\left(\omega ; \gamma_{D}\right)
$$

on the cross section. Here $\nabla_{y}$ is the gradient operator in the coordinates $y$; in what follows $\Delta_{y}$ will stand for the corresponding Laplace operator. The first eigenfunction, normalized in $L_{2}(\omega)$, will be denoted by $U_{\dagger}$; by the maximum principle, we may assume that it is positive.

Theorem 3.1. 1) Under the condition

$$
\int_{\omega} H(y) F_{\dagger}(y) d y<0,
$$

where

$$
F_{\dagger}(y)=\left|\nabla_{y} U_{\dagger}(y)\right|^{2}-\lambda_{\dagger}\left|U_{\dagger}(y)\right|^{2},
$$

the operator $A^{H}$ has an eigenvalue on the interval $\left(0, \Lambda_{\dagger}\right)$, i.e., its discrete spectrum is nonempty.

2) If the integral in (3.4) is zero, but there is a set $\varsigma \subset \omega^{H}$ of positive measure on which the derivative $\partial_{\nu} U_{\dagger}$ differs from zero almost everywhere, then still $\sigma_{d}^{H} \neq \varnothing$. 
Proof. 1) In the minimal principle (1.19), we plug the test function (1.20). Repeating the calculations that led us in Subsection 2 of $\S 2$ to formula (1.22), for the lower bound of the spectrum of the operator $A^{H}$ we obtain the inequality

$$
\underline{\sigma}^{H} \leq \lambda_{\dagger}\left(1+2 \varepsilon \lambda_{\dagger}^{-1} \int_{\omega} H(y)\left(\left|\nabla_{y} U_{\dagger}(y)\right|^{2}-\lambda_{\dagger}\left|U_{\dagger}(y)\right|^{2}\right) d y+O\left(\varepsilon^{2}\right)\right),
$$

which ensures the first claim in the theorem.

2) If we suppose that identities (1.24) are valid and

$$
\int_{\omega} H(y)\left(\left|\nabla_{y} U_{\dagger}(y)\right|^{2}-\lambda_{\dagger}\left|U_{\dagger}(y)\right|^{2}\right) d y=0
$$

then, as in the proof of Theorem 1.1, 2), the test function $u^{\varepsilon}+\sqrt{\varepsilon} v$ will satisfy relation (1.25), which turns into the formula

$$
-2 \sqrt{\varepsilon} \int_{\omega^{H}} v(x) \partial_{\nu} U_{\dagger}(y) d s_{x} \leq C \varepsilon
$$

similar to (1.27). Under the assumptions of the theorem, this leads to a contradiction with (1.24). Consequently, $\underline{\sigma}<\lambda_{\dagger}$ and the discrete spectrum is nonempty.

The integral identity (3.3) shows that expression (3.5) has zero mean over the domain $\omega$. This expression is positive near the part $\gamma_{D}$ of $\partial \omega$ and negative in a neighborhood of the point of maximum of the eigenfunction $U_{\dagger}$. Therefore, it is not hard to construct examples of the profile function $H$ with property (3.4), which ensures the existence of eigenvalues below the threshold $\Lambda_{\dagger}$ of the continuous spectrum.

Let $H \in C^{1}(\bar{\omega})$, and let $\Delta_{y} H \in L_{\infty}(\omega)$. Observe that $U_{\dagger} \in H^{1}(\omega) \subset L_{2}(\partial \omega)$. Substituting the test function $W=H U_{\dagger} \in \stackrel{\circ}{H}^{1}\left(\omega ; \gamma_{D}\right)$ in the integral identity (3.2), we obtain

$$
\begin{array}{r}
\left(H \nabla_{y} U_{\dagger}, \nabla_{y} U_{\dagger}\right)_{\omega}-\lambda_{\dagger}\left(H U_{\dagger}, U_{\dagger}\right)_{\omega}=-\left(\nabla_{y} U_{\dagger}, U_{\dagger} \nabla_{y} H\right)_{\omega}=-\frac{1}{2}\left(\nabla_{y} U_{\dagger}^{2}, \nabla_{y} H\right)_{\omega} \\
=\frac{1}{2} \int_{\omega}\left|U_{\dagger}(y)\right|^{2} \Delta_{y} H(y) d y-\frac{1}{2} \int_{\gamma_{N}}\left|U_{\dagger}(y)\right|^{2} \partial_{\nu} H(y) d s_{y} .
\end{array}
$$

Here $\nu=\left(\nu_{1}, \ldots, \nu_{n-1}\right)$ is the outward unit normal to the boundary of $\omega \subset \mathbb{R}^{n-1}$, and $d s_{y}$ is the area element on $\omega$. Thus, in the case of the Dirichlet problem, i.e., for $\gamma_{D}=\partial \omega$ and $\gamma_{N}=\varnothing$, condition (3.4) is fulfilled provided that $H$ is subharmonic.

2. Cranked many-dimensional waveguide; the mixed boundary-value problem. By (3.6), for the linear function $H(y)=k y_{n-1}, k>0$, condition (3.4) is equivalent to

$$
\int_{\gamma_{N}} U_{\dagger}(y)^{2} \nu_{n-1}(y) d s_{y}>0
$$

In particular, if on the part $\gamma_{N}$ of $\partial \omega$ the projection of the outward normal to the axis $y_{n-1}$ is positive, then problem (3.2) has an eigenvalue on the interval $\left(0, \Lambda_{\dagger}\right)$. We use this observation to verify a claim similar to Theorem 2.1 in the many-dimensional case.

Suppose the boundary of $\omega$ is smooth, i.e., of class $C^{2, \delta}$. We split the cylinder $\omega \times \mathbb{R}^{n}$ into two parts by the hyperplane

$$
\left\{x=\left(y_{1}, \ldots, y_{n-1}, z\right): z=y_{n-1} \tan (\alpha / 2)\right\}, \quad \alpha \in(0, \pi / 2),
$$

and choose the part $\Pi_{1}$ that, for $R$ large, contains the semicylinder $\omega \times(R,+\infty)$. Accordingly, $\Gamma_{1 N}=\partial \Pi_{1} \cap\left(\gamma_{N} \times \mathbb{R}\right)$. The mirror images of $\Pi_{1}$ and $\Gamma_{1 N}$ relative to the hyperplane (3.8) are denoted by $\Pi_{2}$ and $\Gamma_{2 N}$. Put

$$
\bar{\Omega}=\overline{\Pi_{1}} \cup \overline{\Pi_{2}}, \quad \Gamma_{D}=\partial \Omega \backslash\left(\overline{\Gamma_{1 N}} \cup \overline{\Gamma_{2 N}}\right) .
$$


To check our next statement, we can argue as in Subsection 1 of $\S 2$.

Theorem 3.2. If inequality (3.7) is fulfilled, then the discrete spectrum of problem (1.6) in the cranked waveguide $\Omega$ described above cannot be empty.

The sufficient condition (3.7) can be ensured by taking as $\gamma_{N}$ any small neighborhood of a point where the normal $\nu$ coincides with the unit vector of the $y_{n-1}$-axis. In the constructions in Subsection 2 of $\S 1$ and Subsection 1 of $\S 2$, we acted precisely in this way.

3. Cranked many-dimensional waveguide; the Dirichlet problem. Under the boundary conditions (1.4), i.e., for $\gamma_{D}=\partial \omega, \gamma_{N}=\varnothing$, expression (3.6) with a linear function $H$ vanishes for each of the semicylinders with slanted ends that form the cranked waveguide in question. Therefore, the trick 3 employing the test function $u^{\varepsilon}+\sqrt{\varepsilon} v$ (see (1.25) -(1.27) and (2.8)-(2.11) ) results in the following statement.

Theorem 3.3. The discrete spectrum of the operator A corresponding to the Dirichlet problem (1.4) in the cranked many-dimensional waveguide occurring in Theorem 3.2 can never be empty.

4. Branching many-dimensional waveguide; the Dirichlet problem. Suppose $\partial \omega$ is smooth as before, but the profile function $H$ (see (3.1)) is continuous and piecewise linear. For instance, the cross section $\omega$ of the semicylinder $\Pi^{H}$ is split by the hyperplane $\left\{y: y_{n-1}=0\right\}$ into two nonempty parts $\omega_{ \pm}=\left\{y: \pm y_{n-1}>0\right\}$, and

$$
H(y)= \begin{cases}k_{+} y_{1}+k_{0} & \text { if } y \in \omega_{+}, \\ k_{-} y_{1}+k_{0} & \text { if } y \in \omega_{-},\end{cases}
$$

where $k_{ \pm}$and $k_{0}$ are constants (Figure 18a). We turn to the mixed boundary-value problem (3.2), posing the Neumann boundary conditions only on the end $\omega^{H}$ of the semicylinder (3.1). The corresponding model problem (3.3) acquires the Dirichlet boundary conditions on the entire surface $\partial \omega$, i.e., $\gamma_{D}=\partial \omega$ and $\gamma_{N}=\varnothing$, while the sufficient condition (3.4) for the discrete spectrum of problem (3.3) to be nonempty turns by (3.6) into the inequality

$$
\left(k_{+}-k_{-}\right) \int_{v}\left|U_{\dagger}(y)\right|^{2} d s_{y}<0,
$$

where $v=\left\{y \in \omega: y_{n-1}=0\right\}$. Thus, if $k_{+}>k_{-}$, i.e., if the profile function (3.9) is convex, inequality (3.4) is fulfilled. Any convex, continuous, piecewise linear function is subharmonic. This observation together with formulas (3.4) and (3.6) (the latter needs a correct interpretation) leads to the next claim.

Theorem 3.4. If the continuous piecewise linear profile function $H$ (see the definition (3.1) of the semicylinder $\Pi^{H}$ ) is convex, then the discrete spectrum of the operator of the mixed boundary-value problem (3.2) with Neumann conditions on the end and Dirichlet conditions on the lateral surface of $\omega^{H}$ is nonempty.

As in $\S 2$, Theorem 3.4 can be used for finding eigenvalues $\lambda \in\left(0, \lambda_{\dagger}\right)$ of the Dirichlet problem for junctions of complicated nature. For better visibility, we restrict ourselves to discussing 3-dimensional waveguides. If a domain $\omega \subset \mathbb{R}^{2}$ is symmetric with respect to the line $\left\{y=\left(y_{1}, y_{2}\right): y_{2}=0\right\}$, then from several identical prisms with rectangular cross sections and pointed ends (Figure 18a) we can easily build a branching "planar" waveguide (Figure 19a) with nonempty discrete spectrum. In the case of a rich symmetry of the cross section $\omega$, from "pointed stakes" as in Figure 18b we can construct a "really spatial" junction with the desired properties (Figure 19b). Prisms with square cross section can also be used. 
a)

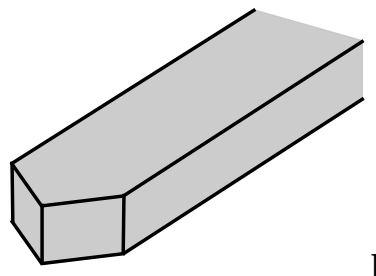

b)

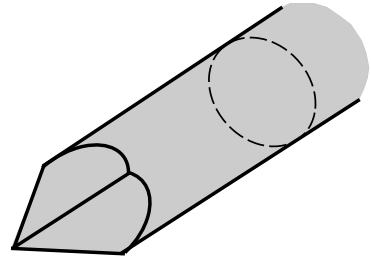

Figure 18. Semicylinders with pointed ends.

a)

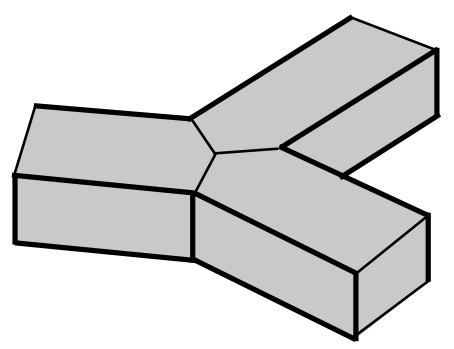

b)

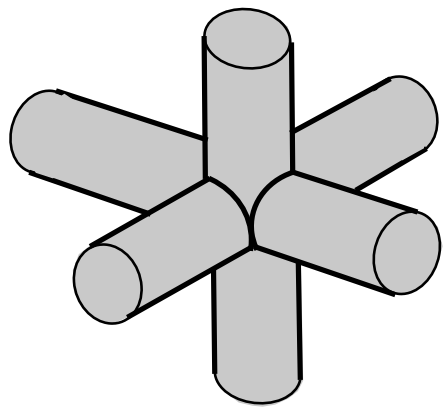

FiguRE 19. Branching 3-dimensional waveguides.

5. Neumann boundary conditions. Suppose that a cranked or branching manydimensional $(n \geq 3)$ waveguide $\Omega$ formed by slanted or pointed semicylinders with identical normal cross sections is symmetric relative to the plane $\mathbb{R}_{n}^{n-1}=\left\{x=\left(x_{1}, \ldots, x_{n}\right)\right.$ : $\left.x_{1}=0\right\}$, and that this plane intersects each 4 of the pointed semicylinders $\Pi_{1}, \ldots, \Pi_{N}$ (see Figure 19a; the waveguide in Figure 19b does not possess the latter property). Consider the upper half $\Omega^{+}=\left\{x \in \Omega: x_{n}>0\right\}$ of $\Omega$; following the approach of [7], on the specially formed surface $\Upsilon=\left\{x \in \Omega: x_{n}=0\right\}$ we pose the Dirichlet boundary conditions, i.e., we consider the mixed boundary-value problem (1.1), (1.5) in the domain $\Omega^{+}$the boundary $\partial \Omega^{+}$of which is split into two parts $\Gamma_{D}=\Upsilon$ and $\Gamma_{N}=\partial \Omega_{+} \backslash \bar{\Upsilon}=(\partial \Omega)^{+}$.

It is important to note that the continuous spectrum of the Neumann problem coincides with the closed real half-axis $\overline{\mathbb{R}_{+}}=[0,+\infty)$, but the mixed boundary-value problem constructed above has a positive threshold $\lambda_{\dagger}^{+}$of the continuous spectrum $\sigma_{c}^{+}\left[\lambda_{\dagger}^{+},+\infty\right)$. The basic idea of the method of [7] consists precisely in creating a positive threshold, and the subsequent study of the discrete spectrum of the corresponding boundary-value problem formed artificially (see also [9, 13] in connection with problems of elasticity theory).

The threshold $\lambda_{\dagger}^{+}$is the first eigenvalue $\Lambda_{\dagger}^{+}$of problem (3.3) in the domain $\omega^{+}=$ $\left\{y \in \omega: y_{1}>0\right\}$ with the Dirichlet boundary condition on the union of the sets $\gamma_{D}^{+}$ and $v=\left\{y \in \omega: y_{1}=0\right\}$. The corresponding eigenfunction will be denoted by $U_{\dagger}^{+}$; we introduce the function $F_{+}^{+}$by formula (3.5) with obvious modifications.

First, we consider problem (3.2) in the half $\Pi^{H+}$ of the waveguide (3.1) with the Dirichlet boundary condition on $\Upsilon^{H+}=\left\{x \in \Pi^{H}: x_{1}=0\right\}$ and the Neumann conditions on $\partial \Pi^{H+} \backslash \Upsilon^{H+}$, assuming the profile function $H$ to be even relative to $y_{1}$. The two claims of Theorem 3.1 remain valid, with clear changes in the statements. Thus, under

\footnotetext{
${ }^{4}$ This requirement cannot be satisfied if $n=2$.
} 
the condition

$$
\int_{\omega^{+}} H(y) F_{\dagger}^{+}(y) d y<0,
$$

where $F_{\dagger}^{+}$is the expression (3.5) constructed by $U_{\dagger}^{+}$and $\Lambda_{\dagger}^{+}$, the operator $A^{H+}$ of the mixed boundary-value problem (1.1), (1.5) in the domain $\Omega^{+}$has a point $\lambda_{1}^{+} \in\left(0, \lambda_{\dagger}^{+}\right)$ of discrete spectrum. We extend the corresponding eigenfunction $u_{1}^{+} \in \stackrel{\circ}{H}^{1}\left(\Pi^{H+} ; \Upsilon^{H}\right)$ as an odd function to the entire waveguide (3.1). Since the Dirichlet conditions are posed on the artificially created surface $\Upsilon^{H}=\left\{x \in \Pi^{H}: y_{1}=0\right\}$, the odd extension turns out to be smooth inside the waveguide, i.e., it is a nontrivial solution of the Neumann problem for the Helmholtz operator $-\Delta_{x}-\lambda_{1}^{+}$in $\Pi^{H}$. In other words, $\lambda_{1}^{+}$is an eigenvalue of the operator $A^{H}$ lying in its point spectrum $\sigma_{p}^{H}$. If inequality (3.10) is replaced by the corresponding identity, then we can apply the now usual trick involving the test function $u^{\varepsilon}+\sqrt{\varepsilon} v$; this leads to the following result.

Theorem 3.5. 1) Under condition (3.10), the operator $A^{H}$ of the Neumann problem in the semicylinder (3.1) has an eigenvalue on the continuous spectrum $[0 .+\infty)$, i.e., its point spectrum is nonempty.

2) If the integral in (3.10) vanishes, but there is a set $\varsigma \subset \omega^{H+}$ of positive measure on which the normal derivative $\partial_{\nu} U_{\dagger}$ is nonzero almost everywhere, then $\sigma_{p}^{H} \neq \varnothing$ as before.

We return to discussing the Neumann problem in a cranked or branching waveguide. For the half $\Pi_{p}^{+}$of each of the semicylinders $\Pi_{p}$, formula (3.6) remains valid with clear modifications, but we cannot directly find the sign of the last integral in (3.6) (recall that in Subsection 1 of $\S 3$ the zone of the Neumann boundary conditions was coordinated with the form of the cross section $\omega$, and that was the only reason why the integral mentioned became positive). Therefore, we can use Theorem 3.5 for finding the point spectrum of the Neumann problem in an arbitrary symmetric cranked or branding waveguide only if the first eigenfunction $U_{1}^{+}$of the mixed boundary-value problem on the half $\omega^{+}$of the cross section is known to a large extent.

Consider the waveguide in Figure 19a, formed by pointed 3-dimensional prisms (as in Figure 18a), with the rectangular cross section $\omega=\left\{y=\left(y_{1}, y_{2}\right):\left|y_{1}\right|<l,\left|y_{2}\right|<1\right\}$. Then

whence

$$
U_{\dagger}^{+}(y)=\sin \left(\frac{\pi}{2} y_{2}\right), \quad \lambda_{\dagger}^{+}=\Lambda_{1}^{+}=\frac{\pi^{2}}{4}, \quad F_{1}^{+}(y)=\frac{\pi^{2}}{4} \cos \left(\pi y_{2}\right),
$$

$$
\int_{0}^{1} F_{1}^{+}\left(y_{1}, y_{2}\right) d y_{2}=0 \text { for any } y_{1} \text {. }
$$

Thus, the condition required in the preceding paragraph is violated: the integral corresponding to each of the half-prisms is zero. Theorem 3.5 (2) also does not allow us to find a point of the discrete spectrum, because on the ends of the half-prisms the derivative of $U_{\dagger}^{+}$along the normal vanishes (the normal has projection $\nu=0$ to the $y_{2}$-axis).

Remark 3.1. The planar branching waveguides as in Figures $2 \mathrm{a}$ and $3 \mathrm{a}$ with the angles $\alpha_{k}=2(k-1) \pi / 3$ and $\alpha_{k}=(k-1) \pi / 2$ (respectively) can be obtained by repeated mirror reflection of cranked waveguides with width $1 / 2$ and angle $\alpha_{2}=2 \pi / 3$ or $\alpha_{2}=\pi / 2$. Unfortunately, since Theorem 2.2 ensures the absence of the discrete spectrum in the case where the Neumann conditions are posed on the "inner" part of the boundary and the Dirichlet conditions are posed on the "exterior" part, the trick of [7] does not yield any eigenvalue of the Neumann problem in such waveguides. If we interchange the location of the boundary conditions on the sides of cranked waveguides (Figure 16), then by 
a)

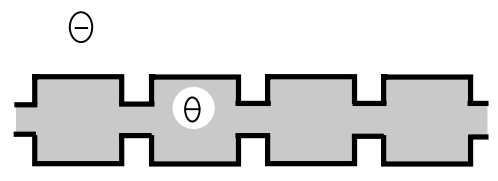

b)

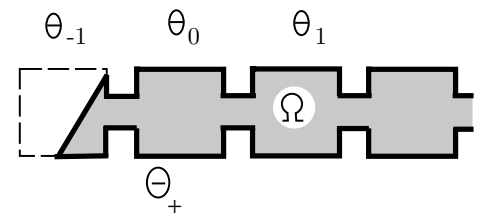

Figure 20. A quasicylinder and a semibounded periodic waveguide.

Theorem 1.1, a discrete spectrum point will arise, and the even extension of the corresponding eigenfunction will lead to an eigenfunction for the first eigenvalue $\lambda_{1} \in\left(0, \pi^{2}\right)$ for branching waveguides with the Dirichlet condition; this was already established in Theorem 1.1. The points of the discrete spectrum for the Dirichlet problems in cranked waveguides (Figure 1c) and the odd extensions of the corresponding eigenfunctions guarantee the presence of an element $\lambda_{\text {}}$ of the point spectrum different from $\lambda_{1}$ (the eigenfunction constructed in this way changes its sign inside the branching waveguide). Since the first positive eigenvalue of the Neumann problem in the unit squarf5 is equal to $\pi^{2}$, the arguments of Example 2.1 prove the absence of the second eigenvalue in the discrete spectrum of a cross-like waveguide, i.e., $\lambda_{\bullet}$ is a point of the continuous spectrum.

\section{$\S 4$. Periodic WAVEguides}

1. Spectrum of a periodic waveguide. Let $\theta$ be a bounded open set in the layer $\{x=(y, z): z \in(0,1)\}$, and let $\Theta$ be the domain with periodic cross section (a quasicylinder, see Figure 20a) composed of the periodicity cells $\theta_{j}=\{x:(y, z-j) \in \theta\}$, $j \in \mathbb{Z}$, i.e., its closure is given by the formula

$$
\bar{\Theta}=\bigcup_{j \in \mathbb{Z}} \overline{\theta_{j}} .
$$

We suppose that the boundary $\partial \Theta$ is an $(n-1)$-dimensional Lipschitz surface and, in particular, the quasicylinder is connected (otherwise $\Theta$ cannot be a domain). Let $\Omega$ be yet another Lipschitz domain such that

$$
\Theta_{+}:=\{x \in \Theta: z>0\} \subset \Omega \subset\{x \in \Theta: z>-1\}
$$

(cf. Figure 20b). In other words, the semiinfinite waveguide $\Omega$ includes the cells $\theta_{0}, \theta_{1}, \theta_{2}, \ldots$, and also a part of the cell $\theta_{-1}$. In the domain $\Omega$ we consider the mixed boundary-value problem (1.1), (1.4).

Unlike the cylindrical waveguides and their combinations treated in the preceding sections and having the ray (1.7) as the continuous spectrum, for a periodic waveguide we have a "band-gap" structure of the essential6 spectrum $\sigma_{e}$, which is the union of infinitely many segments $\Upsilon_{k}, k \in \mathbb{N}$,

$$
\sigma_{e}=\bigcup_{k \in \mathbb{N}} \Upsilon_{k}, \quad \Upsilon_{k}=\left\{\lambda=\Lambda_{k}(\eta) \mid \eta \in[0,2 \pi)\right\} .
$$

Here, $\Lambda_{k}(\eta)$ is an element of the sequence

$$
\Lambda_{1}(\eta), \leq \Lambda_{2}(\eta) \leq \cdots \leq \Lambda_{k}(\eta) \leq \cdots \rightarrow+\infty
$$

\footnotetext{
${ }^{5}$ Unfortunately, in the book 24 the author has not found any formula for the first positive eigenvalue for the Neumann problem in the isosceles triangle and therefore, was unable to make a similar conclusion for the waveguides with the angles $2 \pi / 3$ in Figure $2 \mathrm{a}$.

${ }^{6}$ The author does not know whether a problem in the quasicylinder $\Theta$ can have an eigenvalue of infinite multiplicity. If there are no such numbers (none of the segments $\Upsilon_{k}$ degenerates to a point), then the essential spectrum coincides with the continuous one.
} 
of eigenvalues of the following model problem on the periodicity cell

$$
\begin{gathered}
-\Delta_{x} u(x ; \eta)=\Lambda(\eta) U(x ; \eta), \quad x \in \theta, \\
U(x ; \eta)=0, \quad x \in \partial \theta \backslash\left(\epsilon_{0} \cup \epsilon_{1}\right), \\
U(y, 0 ; \eta)=\exp (i \eta) U(y, 1 ; \eta), \\
\partial_{z} U(y, 0 ; \eta)=\exp (i \eta) \partial_{z} U(y, 1 ; \eta), \quad(y, 0) \in \epsilon_{0} .
\end{gathered}
$$

Note that the quasiperiodicity conditions (4.6) are posed on the "ends" $\epsilon_{0}=\{x \in \partial \theta$ : $z=0\}$ and $\epsilon_{1}=\{x \in \partial \theta: z=1\}$ of the cell. Problem (4.4)-(4.6) is obtained from the Dirichlet problem on the quasicylinder $\Theta$ via the Gelfand transformation [27]

$$
u(y, z) \mapsto U(y, z ; \eta)=\frac{1}{\sqrt{2 \pi}} \sum_{j \in \mathbb{Z}} \exp (-i j \eta) u(y, z-j)
$$

(for the properties of this transformation, see, e.g., [26] [29]). Observe that $(y, z) \in \Theta$ on the left-hand side of (4.1), while $(y, z) \in \theta$ on the right-hand side.

As an ingredient of the integral identity

$$
\left(\nabla_{x} U, \nabla_{x} W\right)_{\theta}=\Lambda(U, W)_{\theta}, \quad W \in \stackrel{\circ}{H}_{\eta-p e r}^{1}\left(\theta ; \partial \theta \backslash\left(\epsilon_{0} \cup \epsilon_{1}\right)\right),
$$

which serves problem (4.4)-(4.6), we have the subspace of functions of the Sobolev class $H^{1}(\theta)$ that satisfy the Dirichlet condition (4.5) and the first quasiperiodicity condition in (4.6). Since the embedding $H^{1}(\theta) \subset L_{2}(\theta)$ is compact (the Lipschitz domain $\theta$ is bounded) and the sesquilinear form on the left in (4.7) is positive, the spectrum of the model problem turns out to be discrete, forming an unboundedly increasing positive sequence (4.3) of eigenvalus. The functions $\eta \mapsto \Lambda_{k}(\eta)$ are continuous (see [30, Chapter 9]) and $2 \pi$-periodic (for obvious reasons). Therefore, the sets $\Upsilon_{k}$ (see (4.2)) are indeed closed and connected segments.

The band-gap structure (4.2) of the spectrum implies that some lacunas may arise, i.e., some intervals having ends in the essential spectrum $\sigma_{e}$ but only containing points of the discrete spectrum $\sigma_{d}$ (see the surveys [27, 31, 21]). However, like in the preceding sections, here our goal is the search of eigenvalues below the threshold

$$
\lambda_{\dagger}=\min \left\{\Lambda_{1}(\eta) \mid \eta \in[0,2 \pi)\right\}
$$

of the essential spectrum. Due to the Dirichlet boundary conditions, the threshold (4.8) is positive. It is also known that the minimum in (4.8) is attained at the point $\eta=0$, but this observation, in essence, will never be used in what follows (see Remark 4.1).

2. Extension of the variational method. Let $\lambda_{\dagger}=\Lambda_{1}(0)$ and $U_{\dagger}(x)=U_{1}(x ; 0)$ be an eigenvalue and an eigenfunction of problem (4.7) (or problem (4.4)-(4.6)) for $\eta=0$. The function $z \mapsto U_{\dagger}(y, z)$ is 1-periodic; it can be chosen to be real and positive in the cell $\theta$. As in (1.20), we put

$$
u^{\varepsilon}(x)= \begin{cases}U_{\dagger}(y, z) & \text { if } z<0 \\ \exp (-\varepsilon z) U_{\dagger}(y, z) & \text { if } z \geq 0\end{cases}
$$

and plug this test function into formula (1.19) for the lower bound of the spectrum of the operators $A$ corresponding to problem (1.1), (1.4) (cf. Subsection 1 of $\S 2$ ) in the domain 
$\Omega$ in Figure 11b. We have

$$
\begin{aligned}
& \left\|u^{\varepsilon} ; L_{2}(\Omega)\right\|^{2}=\left\|u^{\varepsilon} ; L_{2}\left(\Omega \backslash \Theta^{+}\right)\right\|^{2}+\left\|u^{\varepsilon} ; L_{2}\left(\Theta^{+}\right)\right\|^{2} \\
& \left\|u^{\varepsilon} ; L_{2}\left(\Omega \backslash \Theta^{+}\right)\right\|^{2}=\left\|U_{\dagger} ; L_{2}\left(\Omega \backslash \Theta^{+}\right)\right\|^{2} \\
& \left\|u^{\varepsilon} ; L_{2}\left(\Theta^{+}\right)\right\|^{2}=\sum_{j=0}^{\infty} \int_{\theta_{j}} \exp (-2 \varepsilon z)\left|U_{\dagger}(y, z)\right|^{2} d y d z \\
& \quad=\sum_{j=0}^{\infty} \exp (-2 \varepsilon j)\left(\left\|U_{\dagger} ; L_{2}(\theta)\right\|^{2}-2 \varepsilon \int_{\theta_{j}}\left|U_{\dagger}(y, \zeta)\right|^{2} d y d \zeta+O\left(\varepsilon^{2}\right)\right) .
\end{aligned}
$$

We have used the Taylor formula

$$
\exp (-2 \varepsilon(z-j))=\exp (-2 \varepsilon \zeta)=1-2 \varepsilon \zeta+O\left(\varepsilon^{2}\right), \quad \zeta \in(0,1)
$$

Making a similar transformation, we get

$$
\begin{aligned}
& \left\|\nabla_{x} u^{\varepsilon} ; L_{2}(\Omega)\right\|^{2}=\left\|\nabla_{x} u^{\varepsilon} ; L_{2}\left(\Omega \backslash \Theta^{+}\right)\right\|^{2}+\left\|\nabla_{x} u^{\varepsilon} ; L_{2}\left(\Theta^{+}\right)\right\|^{2} \\
& \left\|\nabla_{x} u^{\varepsilon} ; L_{2}\left(\Omega \backslash \Theta^{+}\right)\right\|^{2}=\left\|\nabla_{x} U_{\dagger} ; L_{2}\left(\Omega \backslash \Theta^{+}\right)\right\|^{2} \\
& \left\|u^{\varepsilon} ; L_{2}\left(\Theta^{+}\right)\right\|^{2}=\sum_{j=0}^{\infty} \int_{\theta_{j}} \exp (-2 \varepsilon z)\left(\left|\nabla_{x} U_{\dagger}(y, z)\right|^{2}\right. \\
& \left.\quad+2 \varepsilon U_{\dagger}(y, z) \partial_{z} U_{\dagger}(y, z)+\varepsilon^{2}\left|U_{\dagger}(y, z)\right|^{2}\right) d y d z \\
& =\sum_{j=0}^{\infty} \exp (-2 \varepsilon j)\left(\left\|\nabla_{x} U_{\dagger} ; L_{2}(\theta)\right\|^{2}\right. \\
& \left.\quad-2 \varepsilon \int_{\theta_{j}}\left(\zeta\left|U_{\dagger}(y, \zeta)\right|^{2}-U_{\dagger}(y, \zeta) \partial_{\zeta} U_{\dagger}(y, \zeta)\right) d y d \zeta+O\left(\varepsilon^{2}\right)\right) .
\end{aligned}
$$

Finally, we observe that, first,

$$
\begin{aligned}
\mathcal{E} & :=\sum_{j=0}^{\infty} \exp (-2 \varepsilon j)=\frac{1}{2 \varepsilon}+\sum_{j=0}^{\infty} \int_{j}^{j+1}(\exp (-2 \varepsilon j)-\exp (-2 \varepsilon z) d z \\
& =\frac{1}{2 \varepsilon}+\sum_{j=0}^{\infty} \exp (-2 \varepsilon j)\left(-2 \varepsilon \int_{j}^{j+1}(z-j) d z+O\left(\varepsilon^{2}\right)\right)=\frac{1}{2 \varepsilon}+O(1)
\end{aligned}
$$

and second, by (4.4)-(4.6), for $U=U_{\dagger}$ and $\Lambda=\lambda_{\dagger}$ we have

$$
\begin{aligned}
& \left\|\nabla_{x} U_{\dagger} ; L_{2}(\theta)\right\|^{2}=\lambda_{\dagger}\left\|U_{\dagger} ; L_{2}(\theta)\right\|^{2} \\
& 0=-\int_{\theta} z\left(\Delta_{x} U_{\dagger}(y, z)-\lambda_{\dagger} U_{\dagger}(y, z)\right) U_{\dagger}(y, z) d y d z \\
& \quad=\int_{\theta} z\left(\lambda_{\dagger}\left|U_{\dagger}(y, z)\right|^{2}-\left|\nabla_{x} U_{\dagger}(y, z)\right|^{2}\right) d y d z+\int_{\theta} U_{\dagger}(y, z) \partial_{z} U_{\dagger}(y, z) d y d z .
\end{aligned}
$$


Combining the above formulas, we find that

$$
\begin{aligned}
\underline{\sigma} \leq & \frac{\left\|\nabla_{x} u^{\varepsilon} ; L_{2}(\Omega)\right\|}{\left\|u^{\varepsilon} ; L_{2}(\Omega)\right\|} \\
\leq & \frac{\mathcal{E}\left(\left\|\nabla_{x} U_{\dagger} ; L_{2}(\theta)\right\|^{2}-2 \varepsilon \int_{\theta}\left(z\left|\nabla_{x} U_{\dagger}\right|^{2}-U_{\dagger} \partial_{z} U_{\dagger}\right) d x\right)+\left\|\nabla_{x} U_{\dagger} ; L_{2}\left(\Omega \backslash \Theta^{+}\right)\right\|^{2}+c \varepsilon}{\mathcal{E}\left(\left\|U_{\dagger} ; L_{2}(\theta)\right\|^{2}-2 \varepsilon \int_{\theta} z\left|U_{\dagger}\right|^{2} d x\right)+\left\|U_{\dagger} ; L_{2}\left(\Omega \backslash \Theta^{+}\right)\right\|^{2}-c \varepsilon} \\
\leq & \lambda_{\dagger}\left(1-2 \varepsilon\left\|\nabla_{x} U_{\dagger} ; L_{2}\left(\theta^{+}\right)\right\|^{-2} \int_{\theta}\left(z\left(\left|U_{\dagger}\right|^{2}-\lambda_{\dagger}\left|U_{\dagger}\right|^{2}\right)-U_{\dagger} \partial_{z} U_{\dagger}\right) d x\right. \\
& \left.\quad+2 \varepsilon\left\|\nabla_{x} U_{\dagger} ; L_{2}\left(\theta^{+}\right)\right\|^{-2}\left(\left\|\nabla_{x} U_{\dagger} ; L_{2}\left(\Omega \backslash \Theta^{+}\right)\right\|^{2}-\lambda_{\dagger}\left\|U_{\dagger} ; L_{2}\left(\Omega \backslash \Theta^{+}\right)\right\|^{2}\right)\right)+C \varepsilon^{2} \\
= & \lambda_{\dagger}+2 \varepsilon \lambda_{\dagger}\left\|\nabla_{x} U_{\dagger} ; L_{2}\left(\theta^{+}\right)\right\|^{-2}\left(\left\|\nabla_{x} U_{\dagger} ; L_{2}\left(\Omega \backslash \Theta^{+}\right)\right\|^{2}-\lambda_{\dagger}\left\|U_{\dagger} ; L_{2}\left(\Omega \backslash \Theta^{+}\right)\right\|^{2}\right)+C \varepsilon^{2} .
\end{aligned}
$$

So, we have established the next statement.

Theorem 4.1. Suppose that, for $\eta=0$, the first eigenvalue $\lambda_{\dagger}$ and the corresponding eigenfunction $U_{\dagger}$ of problem (4.7) on the periodicity cell satisfy the inequality

$$
\int_{\Omega \backslash \Theta^{+}} F_{\dagger}(y, z) d y d z<0,
$$

where

$$
F_{\dagger}(y, z)=\left|\nabla_{x} U_{\dagger}(y, z)\right|^{2}-\left|U_{\dagger}(y, z)\right|^{2} .
$$

Then the operator $A$ of the Dirichlet problem (1.1), (1.4) in the domain $\Omega$ with a periodic exit to infinity has an eigenvalue $\lambda \in \sigma_{d}$ lying below the continuous spectrum (see formulas (4.2) and (4.8)).

Remark 4.1. The calculations in (4.10) and (4.11) remain valid if we ignore the relations $\lambda_{\dagger}=\Lambda_{1}(0)$ and $U_{\dagger}(x)=U_{1}(x ; 0)$. In the situation 7 where $\lambda_{\dagger}=\Lambda_{1}\left(\eta_{\dagger}\right), \eta^{\dagger} \in(0,2 \pi)$, and $U_{\dagger}(x)=U_{1}\left(x ; \eta_{\dagger}\right)$, it suffices to replace $U_{\dagger}(y, z)$ in (4.9) by the product $\exp \left(i \eta_{\dagger} j\right) U_{\dagger}(y, z)$ on the cells $\theta_{j}$.

3. Cranked and branching periodic waveguides. By (4.12), the function (4.14) has zero mean over the entire cell $\theta$. Since $F_{\dagger}(x)>0$ for $x \in \partial \theta \backslash\left(\epsilon_{0} \cup \epsilon_{1}\right)$, this function is not identically zero; hence, there exist semibounded periodic waveguides with a nonempty discrete spectrum to the left of its essential spectrum.

If, for some waveguide $\Omega_{+}=\Omega$, the integral in (4.13) happens to be positive, then for the periodic waveguide $\Omega_{-}=\Theta \backslash \bar{\Omega}$, directed oppositely, the sufficient condition (4.13) is fulfilled. The author does not know of any specific quasicylinder $\Theta$ that can be split into two semibounded waveguides $\Omega_{ \pm}$such that each of them has eigenvalues $\lambda_{1}^{ \pm}<\lambda_{\dagger}$ (cf. Remark 1.2). In accordance with Theorem 4.1, the separating surface $\Upsilon$ must possess the following two properties: first, the integral of $F_{\dagger}(x)$ over the part of the cell $\theta$ cut by $\Upsilon$ is zero, and second, the derivative $\partial_{\nu} U_{\dagger}$ is not identically zero.

Whenever the waveguide depicted in Figure 20b satisfies (4.8), the cranked waveguide in Figure 21a also has nonempty discrete spectrum, for the same reasons as in Subsection 1 of $\S 2$. Under easily foreseeable conditions, a similar conclusion can be made also for the branching waveguide of Figure 21b, with the help of the arguments used in Subsection 4 of $\S 2$. Nevertheless, we were not able to make these results unconditional, because the first eigenfunction $U_{\dagger}$ of the model problem (4.4)-(4.6) (or (4.8)) depends on all $n$ spatial variables, and what this function looks like is unknown provided the form of the cell is nontrivial.

\footnotetext{
${ }^{7}$ This situation is impossible for the Helmholtz operator, but can occur, e.g., in the case of the system of elasticity theory equations, for which claims of the type of Theorem 4.1 can be checked along the same lines (see [3] for the case of a domain with half-strips-like exits to infinity).
} 
a)

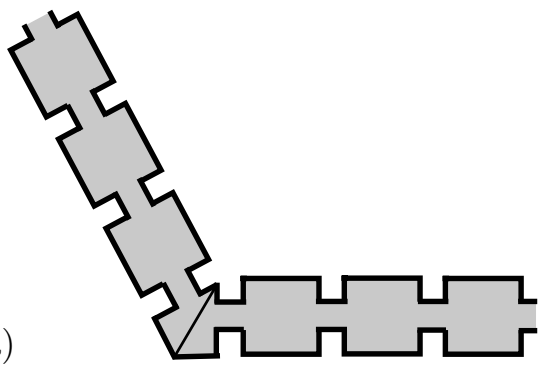

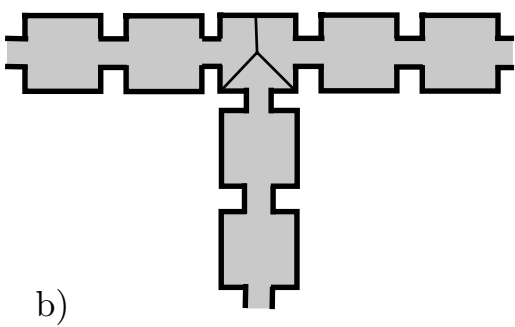

Figure 21. Cranked and branching periodic waveguides.

\section{REFERENCES}

[1] P. Duclos and P. Exner, Curvature-induced bound states in quantum waveguides in two and three dimensions, Rev. Math. Phys. 7 (1995), no. 1, 73-102. MR1310767 (95m:81239)

[2] I. V. Kamotskiı̌ and S. A. Nazarov, Elastic waves localized near periodic families of defects, Dokl. Akad. Nauk 368 (1999), no. 6, 771-773; English transl., Dokl. Phys. 44 (1999), no. 10, 715-717. MR.1749046 (2001c:74043)

[3] Exponentially decreasing solutions of the problem of diffraction by a rigid periodic boundary, Mat. Zametki 73 (2003), no. 1, 138-140; English transl., Math. Notes 73 (2003), no. 1-2, 129-131. MR.1993547

[4] D. S. Jones, The eigenvalues of $\nabla^{2} u+\lambda u=0$ when the boundary conditions are given on semiinfinite domains, Proc. Cambridge Philos. Soc. 49 (1953), 668-684. MR0058086 (15:319c)

[5] F. Ursell, Mathematical aspects of trapping modes in the theory of surface waves, J. Fluid Mech. 183 (1987), 421-437. MR0919481 (89a:76019)

[6] A.-S. Bonnet-Bendhia and F. Starling, Guided waves by electromagnetic gratings and nonuniqueness examples for the diffraction problem, Math. Methods Appl. Sci. 17 (1994), 305-338. MR.1273315 (95c:78008)

[7] D. V. Evans, M. Levitin, and D. Vassiliev, Existence theorems for trapped modes, J. Fluid Mech. 261 (1994), 21-31. MR 1265871 (94m:76113)

[8] S. V. Sukhinin, Waveguide, anomalous, and whispering properties of a periodic chain of obstacles, Sib. Zh. Ind. Mat. 1 (1998), no. 2, 175-198. (Russian) MR1789383 (2001g:78024)

[9] I. Roitberg, D. Vassiliev, and T. Weidl, Edge resonance in an elastic semi-strip, Quart. J. Mech. Appl. Math. 51 (1998), no. 1, 1-13. MR.1610688 (98m:73041)

[10] A.-S. Bonnet-Ben Dhia, J. Duterte, and P. Joly, Mathematical analysis of elastic surface waves in topographic waveguides, Math. Models Methods Appl. Sci. 9 (1999), no. 5, 755-798. MR.1697393 (2000d:74036)

[11] I. V. Kamotskil and S. A. Nazarov, An augmented scattering matrix and exponentially decreasing solutions of an elliptic problem in a cylindrical domain, Zap. Nauchn. Sem. S.-Peterburg. Otdel. Mat. Inst. Steklov. (POMI) 264 (2000), 66-82; English transl., J. Math. Sci. (New York) 111 (2002), no. 4, 3657-3666. MR1796996 (2001m:35246)

[12] S. A. Nazarov, A criterion for the existence of decaying solutions in the problem of a resonator with a cylindrical waveguide, Funktsional. Anal. i Prilozhen. 40 (2006), no. 2, 20-32; English transl., Funct. Anal. Appl. 40 (2006), no. 2, 97-107. MR.2256860 (2007e:35043)

[13] - Trapped modes for a cylindrical elastic waveguide with a damping gasket, Zh. Vychisl. Mat. Mat. Fiz. 48 (2008), no. 5, 863-881; English transl., Comput. Math. Math. Phys. 48 (2008), no. 5, 816-833. MR2433645 (2009e:74061)

[14] I. V. Kamotskiŭ, Surface wave running along the edge of an elastic wedge, Algebra i Analiz 20 (2008), no. 1, 86-92; English transl., St. Petersburg Math. J. 20 (2009), no. 1, 59-63. MR2411969 (2009d:74042)

[15] S. A. Nazarov, A simple method for finding trapped modes in problems of the linear theory of surface waves, Dokl. Akad. Nauk 429 (2009), no. 6, 746-749. (Russian) MR2641034 (2011b:35080)

[16] I. V. Kamotskil and A. P. Kiselev, An energy approach to the proof of the existence of Rayleigh waves in an anisotropic elastic half-space, Prikl. Mat. Mekh. 73 (2009), no. 4, 645-654; English transl., J. Appl. Math. Mech. 73 (2009), no. 4, 464-470. MR2584534 (2011b:74058)

[17] C. M. Linton and P. McIver, Embedded traped modes in water waves and acoustics, Wave Motion 45 (2007), 16-29. MR2441664 (2009e:35216) 
[18] O. A. Ladyzhenskaya, Boundary value problems of mathematical physics, Nauka, Moscow, 1973; English transl., Appl. Math. Sci., vol. 49, Springer-Verlag, New York, 1985. MR 0599579 (58:29032) MR0793735 (87f:35001)

[19] M. Sh. Birman and M. Z. Solomyak, Spectral theory of selfadjoint operators in Hilbert space, Leningrad. Univ., Leningrad, 1980; English transl., D. Reidel Publ. Co., Dordrecht, 1987. MR0609148 (82k:47001) MR1192782 (93g:47001)

[20] I. V. Kamotskiŭ and S. A. Nazarov, On eigenfunctions localized in a neighborhood of the lateral surface of a thin domain, Probl. Mat. Anal., No. 19, Nauchn. Kniga, Novosibirsk, 1999, pp. 105-148; English transl., J. Math. Sci. (New York) 101 (2000), no. 2, 2941-2974. MR1784687 (2001k:35037)

[21] S. A. Nazarov, Properties of spectra of boundary value problems in cylindrical and quasicylindrical domain, Sobolev Spaces in Mathematics. II (V. Maz'ya, ed.), Int. Math. Ser. (N.Y.), vol. 9, Springer, New York, 2009, pp. 261-309. MR2484629 (2009m:35508)

[22] R. L. Shult, D. G. Ravenhall, and H. D. Wyld, Quantum bound states in a classically unbounded system of crossed wires, Phys. Rev. B 39 (1989), no. 8, 5476-5479.

[23] Y. Avishai, D. Bessis, B. G. Giraud, and G. Mantica, Quantum bound states in open geometries, Phys. Rev. B 44 (1991), no. 15, 8028-8034.

[24] G. Pólya and G. Szegő, Isoperimetric inequalities in mathematical physics, Ann. of Math. Stud., No. 27, Princeton Univ. Press, Princeton, NJ, 1951. MR.0043486 (13:270d)

[25] I. M. Gel'fand, Expansion in characteristic functions of an equation with periodic coefficients, Dokl. Akad. Nauk SSSR (N. S.) 73 (1950), no. 6, 1117-1120. (Russian) MR0039154 (12:503a)

[26] S. A. Nazarov, Elliptic boundary value problems with periodic coefficients in a cylinder, Izv. Akad. Nauk SSSR Ser. Mat. 45 (1981), no. 1, 101-112; English transl in Math. USSR-Izv. 18 (1982), no. 1. MR0607578 (82e:35035)

[27] P. A. Kuchment, Floquet theory for partial differential equations, Uspekhi Mat. Nauk 37 (1982), no. 4, 3-52; English transl., Russian Math. Surveys 37 (1982), no. 4, 60-94. MR0667973(84b:35018)

[28] S. A. Nazarov and B. A. Plamenevskiu, Elliptic problems in domains with piecewise smooth boundaries, Nauka, Moscow, 1991; English transl., de Gruyter Exp. Math., vol. 13, Walter de Gruyter, Berlin, 1994. MR 1283387(95h:35001)

[29] P. Kuchment, Floquet theory for partial differential equations, Oper. Theory Adv. Appl., vol. 60, Birkhäuser, Basel, 1993. MR:1232660 (94h:35002)

[30] T. Kato, Perturbation theory for linear operators, 2nd ed., Grundlehren Math. Wiss., Bd. 132, Springer-Verlag, Berlin-New York, 1976. MR0407617 (53:11389)

[31] P. Kuchment, The mathematics of photonic crystals, Mathematical Modeling in Optical Science, Frontiers Appl. Math., vol. 22, SIAM, Philadelphia, 2001, pp. 207-272. MR.1831334 (2002k:78002)

Institute of Mechanical Engineering Problems, Bol'shoŭ Pr. V. O. 61, St. Petersburg 199178, Russia

E-mail address: srgnazarov@yahoo.co.uk

Received 10/OCT/2009

Translated by A. PLOTKIN 